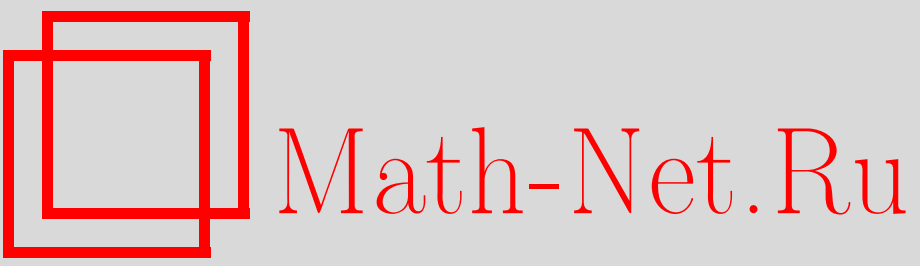

Г. А. Чечкин, Асимптотические разложения собственных значений и собственных функций эллиптического оператора в области с большим количеством близко расположенных на границе "легких" концентрированных масс. Двумерный случай, Изв. РАН. Сер. матем., 2005, том 69, выпуск 4, 161-204

DOI: https://doi.org/10.4213/im652

Использование Общероссийского математического портала Math-Net.Ru подразумевает, что вы прочитали и согласны с пользовательским соглашением

http://www. mathnet.ru/rus/agreement

Параметры загрузки:

IP : 18.208 .226 .222

26 апреля 2023 г., 13:22:41 


\author{
Г. А. Чечкин
}

\title{
Асимптотические разложения собственных значений и собственных функций эллиптического оператора в области с большим количеством близко расположенных на границе "легких" концентрированных масс. Двумерный случай
}

\footnotetext{
Рассмотрены колебания мембраны, содержащей большое количество концентрированных масс на границе. Исследовано асимптотическое поведение частот собственных колебаний такой мембраны при стремлении малого параметра, характеризующего диаметр и плотность концентрированных масс, к нулю. Построены асимптотические разложения собственных элементов соответствующих задач и разложения аккуратно обоснованы.

Библиография: 73 наименования.
}

\section{Введение}

Поведение тел с неоднородной плотностью достаточно сложное, и его изучение представляется интересной задачей, которая не могла быть успешно решена без использования соответствующего математического аппарата. С появлением такого аппарата в конще XIX - начале XX веков начинается активное развитие теории и методов решения таких задач.

Первая математическая работа [1], положившая начало глубоким исследованиям в этой области, выходит в 1913 г. В ней А. Н. Крылов рассматривает задачу о колебаниях струны, нагруженной сосредоточенными массами. В [2, приложение к гл. 2] изучаются собственные частоты колебаний струны, нагруженной сосредоточенной массой в одной точке, рассматривается предельное поведение решений задачи при стремлении массы к нулю и бесконечности. К сожалению, в формулах этой работы есть неточности.

В работе [3] впервые рассмотрена задача, в которой сосредоточенная масса сконцентрирована в $\varepsilon$-окрестности внутренней точки, $\varepsilon$ - мальй параметр, описывающий конщентрацию и размер массы. В этой работе были использованы методы спектральной теории возмущений.

Другой подход был предложен в работах [4]-[8], где вводится новый основной параметр колебательных систем с локально присоединенными массами - отношение присоединенной массы к массе всей системы. При этом удалось описать локальные колебания системы вблизи сосредоточенной массы. Анализ размерностей в задаче

Работа выполнена при частичной поддержке РФФИ (грант № 02-01-00693). 
о спектральных свойствах колебательных систем с конщентрированными массами впервые сделан в [9]. В работе [3] рассмотрена аналогичная задача для оператора Лапласа с граничными условиями Дирихле в трехмерном случае. В [5]-[8] это сделано для всех размерностей и произвольных масс.

В работе [10] исследован случай одной концентрированной массы для одномерного оператора с граничным условием Дирихле. Случай конечного числа концентрированных масс был рассмотрен в [11]. Задача о колебаниях упругого стержня и упругой пластинки с концентрированными массами изучена в работах [12], [13] (см. также [14], [15] и [17]). Случай граничных условий Неймана разобран в [18] и [19]. В [20] исследуются первая и третья краевые задачи для оператора Лапласа в случае, когда плотность возмушена конечным числом конщентрированных масс. Асимптотические разложения собственных значений и собственных функций задачи Дирихле для оператора Лапласа получены в [21] и [22]. Случай трехмерной линейной стационарной системы теории упругости рассмотрен в [23] (см. также [24]). Двумерная задача о колебании мембраны исследована в [25].

В работах [26]-[34] рассматривается асимптотика колебаний тела, имеющего много небольших включений большой плотности, расположенных периодически вдоль гранищы. Изучены вопросы асимптотического поведения собственных значений, т. е. сходимости собственных значений исходной задачи к собственным значениям предельной (усредненной) задачи. Аналогичные задачи были рассмотрены в [35]-[37]. В работе [38] рассматривается краевая задача для стационарной системы линейной теории упругости с непериодическими быстро меняющимися граничными условиями и большим количеством концентрированных масс около границы, асимптотическое поведение ее решений, а также предельное поведение спектра этой краевой задачи. Разобран случай краевой задачи для системы уравнений линейной теории упругости, когда предельная задача имеет третье краевое условие на границе области, а плотность включений не слишком велика. Получены оценки скорости сходимости решения исходной задачи к решению усредненной, а также поведение собственных элементов такой краевой задачи. Следует заметить, что в большинстве работ закон колебания груза или уплотнения описьвается теми же уравнениями, которыми описываются колебания самой системы. В работе [39] рассматривается задача для линейной стационарной системы уравнений теории упругости в областях с концентрированными массами. Рассмотрены различные типы поведения собственных элементов таких задач. В работе [39] рассматривается случай, когда включения достаточно жесткие. При этом законы колебания тела и масс различны. Работа [40] (см. также[41]) посвящена детальному изучению поведения собственных элементов оператора Лапласа в области с непериодическими “легкими” концентрированными массами. В работе [42] (см. также [43]) рассматривается многомерная задача в области с периодическими “легкими" массами. Получены оценки скорости сходимости собственных чисел и собственных функций исходной задачи к соответствуюшим собственным числам и функциям предельной задачи. В [44] анонсированы результаты исследования задач с концентрированными массами в случае "критической" плотности.

В настоящей работе рассматривается двумерная задача в области с периодически расположенными “легкими" массами. Предполагается, что расстояния между 
массами и их диаметр имеют один и тот же порядок малости. Методом согласования асимптотических разложений [66], [70] строятся полные асимптотические разложения собственных значений и собственных функций. Эти разложения обосновываются. Результаты частично анонсированы в [45] и [46].

\section{$\S 1$. Постановка задачи}

Обозначим через $\Omega$ область в $\mathbb{R}^{2}$, лежашую в верхней полуплоскости, граница которой является кусочно гладкой и состоит из нескольких частей: $\partial \Omega=\Gamma_{1} \cup \Gamma_{2} \cup$ $\Gamma_{3} \cup \Gamma_{4}$, при этом $\Gamma_{4}=\Gamma_{\varepsilon} \cup \gamma_{\varepsilon}$, где $\Gamma_{4}$ - отрезок $\left[-\frac{\pi}{2}, \frac{\pi}{2}\right]$ на оси абсцисс, а $\Gamma_{2}$ и $\Gamma_{3}$ принадлежат прямым $x_{1}=-\frac{\pi}{2}$ и $x_{1}=\frac{\pi}{2}$ соответственно. Более того, $\Gamma_{\varepsilon}$ и $\gamma_{\varepsilon}$ имеют микронеоднородную структуру, и их составляющие малые интервалы чередуются между собой (см. рис. 1). Здесь $\varepsilon=\frac{1}{2 \mathcal{N}+1}-$ мальй параметр, $\mathcal{N}$ натуральное число, $\mathcal{N} \gg 1$.

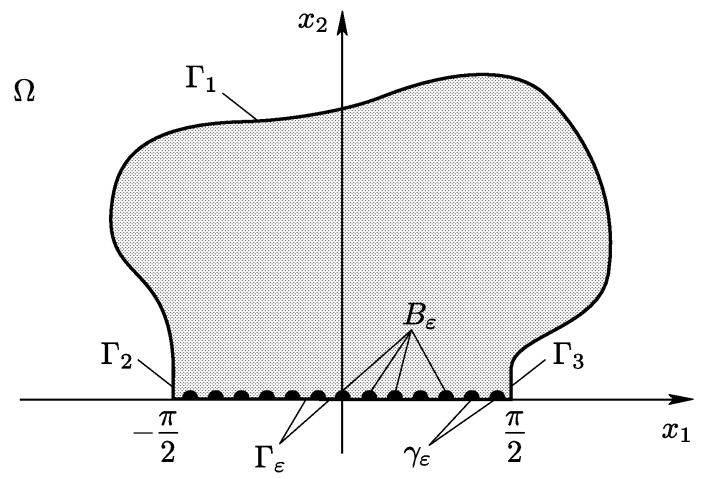

Рис. 1. Мембрана с большим количеством концентрированных масс на границе

Опишем подробнее мелкомасштабную структуру $\Gamma_{4}$. Пусть $\gamma=\{\xi:-a<$ $\left.\xi_{1}<a, \xi_{2}=0\right\}, \Gamma=\left\{\xi:-\frac{\pi}{2}<\xi_{1}<-a, a<\xi_{1}<\frac{\pi}{2}, \xi_{2}=0\right\}$ в переменных $\xi=\frac{x}{\varepsilon}$, при этом $0<a<\frac{\pi}{2}$. Обозначим $\gamma_{\varepsilon}=\left\{x \in \Gamma_{4}: \varepsilon^{-1}\left(x_{1}-j, 0\right) \in \gamma, j \in \mathbb{Z}\right\}$, $\Gamma_{\varepsilon}=\Gamma_{4} \backslash \gamma_{\varepsilon}$.

Будут также использоваться следующие обозначения. Пусть $\Pi=\left\{\xi:-\frac{\pi}{2}<\right.$ $\left.\xi_{1}<\frac{\pi}{2}, \xi_{2}>0\right\}$ - полуполоса, $B$-полукруг $\left\{\xi: \xi_{1}^{2}+\xi_{2}^{2}<a^{2}, \xi_{2}>0\right\}$ (см. рис. 2).

Обозначим $B_{\varepsilon}^{j}=\left\{x \in \Omega: \varepsilon^{-1}\left(x_{1}-j, x_{2}\right) \in B\right\}, j \in \mathbb{Z}, B_{\varepsilon}=\bigcup B_{\varepsilon}^{j}$.

Нашей целью является построение асимптотики при $\varepsilon \rightarrow 0$ собственных элементов следующей спектральной задачи:

$$
\begin{aligned}
-\Delta u_{\varepsilon} & =\lambda_{\varepsilon} \rho_{\varepsilon} u_{\varepsilon} & & \text { в } \quad x \in \Omega, \\
u_{\varepsilon} & =0 & & \text { на } \quad x \in \gamma_{\varepsilon}, \\
\frac{\partial u_{\varepsilon}}{\partial \nu} & =0 & & \text { на } \quad x \in \Gamma_{\varepsilon} \cup \Gamma_{1} \cup \Gamma_{2} \cup \Gamma_{3},
\end{aligned}
$$


где $\rho_{\varepsilon}(x)$ - плотность, имеющая вид

$$
\rho_{\varepsilon}(x)= \begin{cases}1 & \text { в } \Omega \backslash \bar{B}_{\varepsilon}, \\ (a \varepsilon)^{-m} & \text { в } B_{\varepsilon} .\end{cases}
$$

Всюду далее рассматривается постоянная величина $0<m<2$. Будем называть множества $B_{\varepsilon}^{j}$ концентрированными массами.

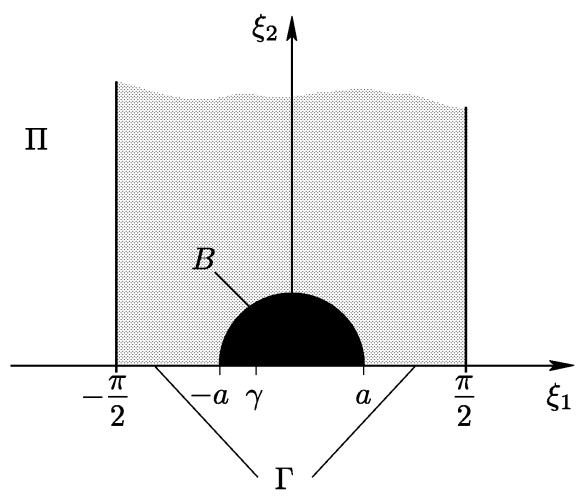

Рис. 2. Ячейка периодичности

Рассмотрим также задачу

$$
\begin{aligned}
-\Delta u_{0}=\lambda u_{0} & \text { в } \quad \Omega, \\
u_{0}=0 & \text { на } \quad \Gamma_{4}, \\
\frac{\partial u_{0}}{\partial \nu}=0 & \text { на } \quad \Gamma_{1} \cup \Gamma_{2} \cup \Gamma_{3} .
\end{aligned}
$$

В следующем параграфе будет показано, что задача (1.3) является предельной для задачи (1.1) (другое доказательство в случае отсутствия масс см. в [47]).

\section{§2. Сходимость и оценки}

Рассмотрим следующую краевую задачу:

$$
\begin{array}{rlrl}
-\Delta U_{\varepsilon} & =\lambda \rho_{\varepsilon} U_{\varepsilon}+f & \text { в } \Omega, \\
U_{\varepsilon} & =0 & & \text { на } \gamma_{\varepsilon}, \\
\frac{\partial U_{\varepsilon}}{\partial \nu} & =0 & & \text { на } \Gamma_{\varepsilon} \cup \Gamma_{1} \cup \Gamma_{2} \cup \Gamma_{3},
\end{array}
$$

где $\rho_{\varepsilon}(x)$ - плотность, имеюшая вид $(1.2)$, и задачу, назьваемую предельной (усредненной):

$$
\begin{aligned}
-\Delta U_{0} & =\lambda U_{0}+f & & \text { в } \Omega, \\
U_{0} & =0 & & \text { на } \Gamma_{4}, \\
\frac{\partial U_{0}}{\partial \nu} & =0 & & \text { на } \Gamma_{1} \cup \Gamma_{2} \cup \Gamma_{3} .
\end{aligned}
$$


Обоснованность такого названия будет показана ниже. Решение задач (1.1), (1.3), (2.1) и (2.2) будем понимать в обобщенном смысле [57] (см. также [60], [61]).

Пусть $f \in L_{2}(\Omega)$. Обозначим через $\|u\|_{0}$ и $\|u\|_{1}$ нормы функции $u$ соответственно в пространствах $L_{2}(\Omega)$ и $H^{1}(\Omega)$.

Целью в этом параграфе является доказательство следующих трех утверждений.

TЕОрема 2.1. Пусть $f \in L_{2}(\Omega), \quad K$ - произвольный компакт на комплексной плоскости $\mathbb{C}$, не содержащий собственных значений предельной задачи (1.3). Тогда:

1) существует число $\varepsilon_{0}>0$ такое, что при любом $\varepsilon<\varepsilon_{0}$ и любом $\lambda \in K$ решение задачи (2.1) существует и единственно, а также справедлива равномерная по в и $\lambda$ оценка

$$
\left\|U_{\varepsilon}\right\|_{1} \leqslant C\|f\|_{0}
$$

где $C$ не зависит также и от $f$;

2) для решений задач (2.1) и (2.2) имеет место сходимость

$$
\left\|U_{\varepsilon}-U_{0}\right\|_{1} \rightarrow 0 \quad \text { npu } \quad \varepsilon \rightarrow 0 .
$$

ТЕОРема 2.2. Пусть $\lambda_{0}$ - собственное значение предельной задачи (1.3). Тогда:

1) существует собственное значение $\lambda_{\varepsilon}$ задачи (1.1), сходящееся $\kappa \lambda_{0}$ при $\varepsilon \rightarrow 0$;

2) если кратность $\lambda_{0}$ равна $N$, то к нему сходится $N$ собственных значений исходной задачи (с учетом совокупной кратности).

Теорема 2.3. Пусть $f \in L_{2}(\Omega)$. Если кратность собственного значения $\lambda_{0}$ предельной задачи (1.3) равна $N$, то:

1) для любого $\lambda$, близкого $\kappa \lambda_{0}$, и решения $U_{\varepsilon}$ краевой задачи (2.1) справедлива равномерная оченка

$$
\left\|U_{\varepsilon}\right\|_{1} \leqslant C \frac{\|f\|_{0}}{\prod_{j=1}^{N}\left|\lambda_{\varepsilon}^{j}-\lambda\right|},
$$

где $\lambda_{\varepsilon}^{1}, \ldots, \lambda_{\varepsilon}^{N}-$ собственные значения задачи (1.1), сходящиеся $\kappa \lambda_{0}$;

2) если решение $U_{\varepsilon}$ задачи (2.1) ортогонально в $L_{2}(\Omega)$ собственной функции $u_{\varepsilon}^{i}$ задачи (1.1), соответствующей $\lambda_{\varepsilon}^{i}$, то имеет место оченка

$$
\left\|U_{\varepsilon}\right\|_{1} \leqslant C \frac{\|f\|_{0}}{\prod_{j=1, j \neq i}^{N}\left|\lambda_{\varepsilon}^{j}-\lambda\right|} .
$$

Доказательство теорем проводится с использованием схемы, приведенной в [48] (см. также [49]-[53]). Необходимо отметить, что наличие концентрированных масс приводит к дополнительным трудностям и мы модифицируем указанную схему. Для этого понадобятся следующие два утверждения.

Обозначим $H^{1}\left(\Omega, \gamma_{\varepsilon}\right)$ пространство функций из соболевского пространства $H^{1}(\Omega)$, имеющих нулевой след на $\gamma_{\varepsilon}$. 
Лемма 2.1. Для последовательности функиий $v_{\varepsilon} \in H^{1}\left(\Omega, \gamma_{\varepsilon}\right)$ имеет место оченка

$$
\int_{B_{\varepsilon}^{i}}\left|v_{\varepsilon}\right|^{2} d x \leqslant C(a \varepsilon)^{2} \int_{B_{\varepsilon}^{i}}\left|\nabla v_{\varepsilon}\right|^{2} d x .
$$

Утверждение верно для любого полукруга $B_{\varepsilon}^{i}$.

ДоказАтельство см., например, в [54, (5.5), с. 263] (см. также [42]).

Обозначим через $H^{1}\left(\Omega, \Gamma_{4}\right)$ пространство функций из $H^{1}(\Omega)$, имеющих нулевой след на $\Gamma_{4}$.

ЛЕмма 2.2. Пусть $v_{\varepsilon}-$ последовательность функций из пространства $H^{1}\left(\Omega, \gamma_{\varepsilon}\right)$ u $v_{\varepsilon} \rightarrow v^{*}$ при $\varepsilon \rightarrow 0$ слабо в $H^{1}(\Omega)$. Тогда $v^{*} \in H^{1}\left(\Omega, \Gamma_{4}\right)$.

ДокАЗАТЕЛЬство. Доказательство проводится с использованием схемы, приведенной в [56].

Рассмотрим полосу $D_{\omega}=\left\{x:-\frac{\pi}{2}<x_{1}<\frac{\pi}{2}, 0<x_{2}<\omega\right\}$. Положим

$$
\begin{aligned}
& \Gamma_{\varepsilon}^{\omega}=\left\{x \in \bar{D}_{\omega}:\left(x_{1}, 0\right) \in \Gamma_{\varepsilon}, x_{2}=\omega\right\}, \\
& \gamma_{\varepsilon}^{\omega}=\left\{x \in \bar{D}_{\omega}:\left(x_{1}, 0\right) \in \gamma_{\varepsilon}, x_{2}=\omega\right\} .
\end{aligned}
$$

Рассмотрим случай $a=\frac{\pi}{4}$. Пусть $D_{\omega}^{1}=\gamma_{\varepsilon} \times(0, \omega)$, а область $D_{\omega}^{2}$ - это объединение параллелограммов, получаюшихся из $D_{\omega}^{1}$ деформацией: фиксируем нижние основания и сдвигаем на $2 a$ вправо верхние так, чтобы они совпали с $\Gamma_{\varepsilon}^{\omega}$ (см. рис. 3 ).

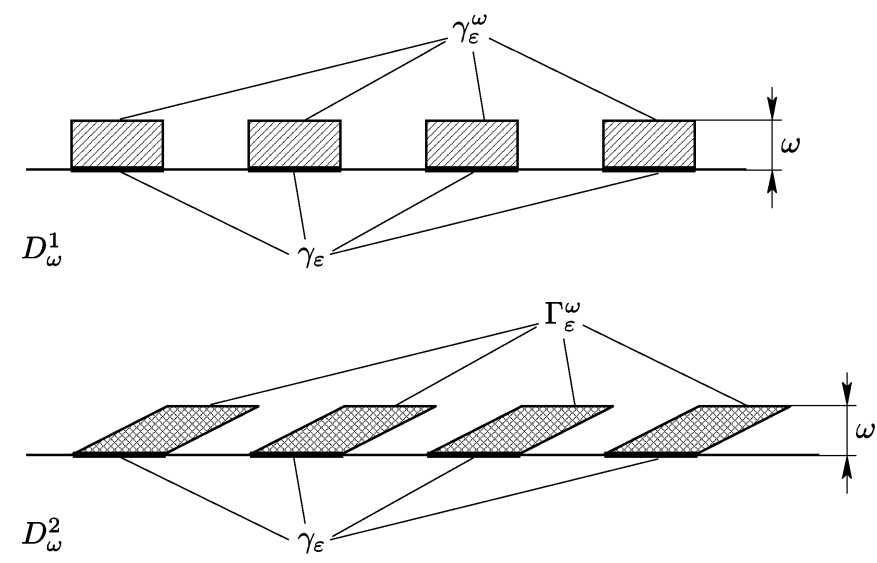

Рис. 3. Прямоугольники и параллелограммы

Имеем

$$
v_{\varepsilon}\left(x_{1}, \omega\right)=\int_{0}^{\omega} \frac{\partial v_{\varepsilon}}{\partial x_{2}} d x_{2},
$$

если $\left(x_{1}, \omega\right) \in \gamma_{\varepsilon}^{\omega}$. Поэтому

$$
v_{\varepsilon}^{2}\left(x_{1}, \omega\right) \leqslant \omega \int_{0}^{\omega}\left(\frac{\partial v_{\varepsilon}}{\partial x_{2}}\right)^{2} d x_{2} .
$$


Проинтегрируем неравенство (2.7) по $\gamma_{\varepsilon}^{\omega}$. Используя формулу Коши-Буняковского, получим

$$
\int_{\gamma_{\varepsilon}^{\omega}} v_{\varepsilon}^{2}\left(x_{1}, \omega\right) d x_{1} \leqslant \omega \int_{\gamma_{\varepsilon}^{\omega}} \int_{0}^{\omega}\left(\frac{\partial v_{\varepsilon}}{\partial x_{2}}\right)^{2} d x_{2} d x_{1} \leqslant \omega \int_{D_{\omega}^{1}}\left|\nabla v_{\varepsilon}\right|^{2} d x .
$$

Оценим теперь $v_{\varepsilon}\left(x_{1}, \omega\right)$ в точке с координатами $\left(x_{1}, \omega\right)$, принадлежащей $\Gamma_{\varepsilon}^{\omega}$. Обозначим эту точку $b_{2}$. Имеем

$$
v_{\varepsilon}\left(x_{1}, \omega\right)=\int_{b_{1}}^{b_{2}} \frac{\partial v_{\varepsilon}}{\partial l} d l
$$

точки $b_{1}$ и $b_{2}$ лежат соответственно на $\gamma_{\varepsilon}$ и $\Gamma_{\varepsilon}^{\omega}$ и принадлежат прямой $l$, параллельной боковой стороне параллелограмма. Отсюда следует неравенство

$$
v_{\varepsilon}^{2}\left(x_{1}, \omega\right) \leqslant \omega \int_{b_{1}}^{b_{2}}\left(\frac{\partial v_{\varepsilon}}{\partial l}\right)^{2} d l
$$

Интегрируя это неравенство по $\Gamma_{\varepsilon}^{\omega}$, получим

$$
\int_{\Gamma_{\varepsilon}^{\omega}} v_{\varepsilon}^{2}\left(x_{1}, \omega\right) d x_{1} \leqslant \omega \int_{\Gamma_{\varepsilon}^{\omega}} \int_{b_{1}}^{b_{2}}\left(\frac{\partial v_{\varepsilon}}{\partial l}\right)^{2} d l d x_{1} \leqslant \omega C \int_{D_{\omega}^{2}}\left|\nabla v_{\varepsilon}\right|^{2} d x
$$

Суммируя неравенства $(2.8)$ и $(2.9)$, имеем

$$
\int_{\Gamma_{4}^{\omega}} v_{\varepsilon}^{2}(x) d x_{1} \leqslant \omega C_{1} \int_{D_{\omega}}\left|\nabla v_{\varepsilon}\right|^{2} d x \leqslant \omega M
$$

где $\Gamma_{4}^{\omega}$ - отрезок, отстоящий от $\Gamma_{4}$ на расстоянии $\omega$, т. е. $\bar{\Gamma}_{4}^{\omega}=\bar{\Gamma}_{\varepsilon}^{\omega} \cup \bar{\gamma}_{\varepsilon}^{\omega}$.

Учитывая компактность вложения $H^{1}\left(\Omega, \gamma_{\varepsilon}\right)$ в $L_{2}\left(\Gamma_{4}\right)$ (см. [57], [58]), перейдем к пределу в неравенстве $(2.10)$ при $\varepsilon \rightarrow 0$, откуда находим

$$
\int_{\Gamma_{4}^{\omega}}\left(v^{*}\right)^{2} d x_{1} \leqslant \omega M
$$

В силу того, что $\omega$ - произвольно малое положительное число и $v^{*} \in H^{1}(\Omega)$, из $(2.11)$ следует, что $v^{*}=0$ на границе $\Gamma_{4}$.

В случае $a>\frac{\pi}{4}$ построения аналогичные, а если $a<\frac{\pi}{4}$, то можно повторить процедуру построения и суммирования несколько раз, строя соответствуюшие параллелограммы (см. рис. 4).

Сначала мы строим первый параллелограмм (нижнее основание совпадает с $\gamma_{\varepsilon}$, а верхнее сдвинуто на $2 a$ вправо). Представляем функцию в виде интеграла, как было описано вьше. Потом строим следующий параллелограмм (сдвиг на 4a). Процедуру повторяем до тех пор, пока весь отрезок $\Gamma_{\varepsilon}^{\omega}$ покроется верхними основаниями параллелограммов. Шагов будет конечное число, так как $а$ фиксировано. Лемма доказана. 


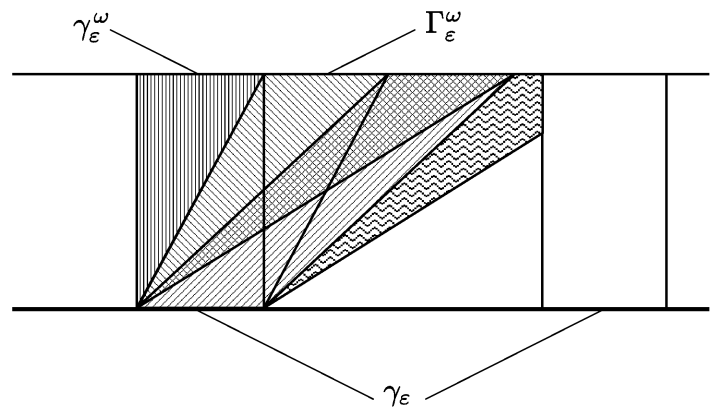

Рис. 4. Параллелограммы в случае $a<\frac{\pi}{4}$

ДОКАЗАТЕЛЬСТво ТЕОРЕмЫ 2.1. 1) НаПомним, что функция $U_{\varepsilon} \in H^{1}\left(\Omega, \gamma_{\varepsilon}\right)$ называется обобшенным решением задачи (2.1), если имеет место интегральное тождество

$$
\int_{\Omega}\left(\nabla U_{\varepsilon}, \nabla \bar{v}\right) d x-\lambda \int_{\Omega} \rho_{\varepsilon} U_{\varepsilon} \bar{v} d x=\int_{\Omega} f \bar{v} d x
$$

для любой $v \in H^{1}\left(\Omega, \gamma_{\varepsilon}\right)$ (аналогично определяются решения задач $(1.1),(1.3)$ и $(2.2))$.

Для доказательства существования и единственности решения достаточно показать, что выполнена оценка (2.3). Действительно, вычтем и добавим член $\int_{\Omega} \rho_{\varepsilon} U_{\varepsilon} \bar{v} d x$ в интегральное тождество $(2.12)$, в результате получим

$$
\int_{\Omega}\left(\left(\nabla U_{\varepsilon}, \nabla \bar{v}\right)+\rho_{\varepsilon} U_{\varepsilon} \bar{v}\right) d x-(\lambda+1) \int_{\Omega} \rho_{\varepsilon} U_{\varepsilon} \bar{v} d x=\int_{\Omega} f \bar{v} d x .
$$

Обозначим через $L_{2, \rho}(\Omega)$ и $H_{\rho}^{1}(\Omega)$ весовые гильбертовы пространства со скалярными произведениями, заданными соответственно формулами

$$
\begin{aligned}
(u, \bar{v})_{0, \rho} & \equiv \int_{\Omega} \rho_{\varepsilon} u \bar{v} d x \\
(u, \bar{v})_{1, \rho} & \equiv \int_{\Omega}\left((\nabla u, \nabla \bar{v})+\rho_{\varepsilon} u \bar{v}\right) d x
\end{aligned}
$$

С учетом обозначений переписьваем (2.13) следуюшим образом:

$$
\left(U_{\varepsilon}, \bar{v}\right)_{1, \rho}-(\lambda+1)\left(U_{\varepsilon}, \bar{v}\right)_{0, \rho}=(f, \bar{v})_{0}
$$

По теореме Рисса (см., например, [55]) существуют оператор $A$ и элемент $F \in$ $H_{\rho}^{1}(\Omega)$ такие, что интегральное тождество переписывается в виде

$$
\left(U_{\varepsilon}, \bar{v}\right)_{1, \rho}-(\lambda+1)\left(A U_{\varepsilon}, \bar{v}\right)_{1, \rho}=(F, \bar{v})_{1, \rho}
$$

Разрешимость этого уравнения следует, например, из тривиальности ядра оператора $[I-(\lambda+1) A]$, которая, в свою очередь, следует из единственности решения однородного уравнения. Единственность решения непосредственно следует из оценки (2.3). 
Подставив в интегральное тождество (2.14) в качестве пробной функции $v=U_{\varepsilon}$, получаем оценку

$$
\begin{aligned}
\left\|U_{\varepsilon}\right\|_{1}^{2} & \leqslant\left\|U_{\varepsilon}\right\|_{1, \rho}^{2} \leqslant C_{1}\left(\|f\|_{0}\left\|U_{\varepsilon}\right\|_{0}+\left\|U_{\varepsilon}\right\|_{0, \rho}^{2}\right) \\
& \leqslant C_{1}\left(\|f\|_{0}\left\|U_{\varepsilon}\right\|_{0}+\left\|U_{\varepsilon}\right\|_{0}^{2}+\varepsilon^{2-m}\left\|U_{\varepsilon}\right\|_{1}^{2}\right) .
\end{aligned}
$$

Здесь мы воспользовались леммой 2.1. При достаточно малом $\varepsilon$ очевидным образом выводим

$$
\left\|U_{\varepsilon}\right\|_{1} \leqslant \frac{C_{1}}{1-C_{1} \varepsilon^{2-m}}\left(\|f\|_{0}+\left\|U_{\varepsilon}\right\|_{0}\right) \leqslant \mathcal{C}\left(\|f\|_{0}+\left\|U_{\varepsilon}\right\|_{0}\right),
$$

где $C_{1}$ и $\mathcal{C}$ не зависят от $\varepsilon, \lambda$ и $f$.

Далее доказательство ведется от противного. Допустим, что оценка (2.3) неверна, т. е. существуют последовательности $\varepsilon_{k} \rightarrow 0$ при $k \rightarrow+\infty, f_{k} \in L_{2}(\Omega)$ и $\lambda_{k}$ такие, что при $\varepsilon=\varepsilon_{k}, \lambda=\lambda_{k}$ и $f=f_{k}$ для решений выполняется обратное неравенство

$$
\left\|U_{\varepsilon_{k}}\right\|_{1}>k\left\|f_{k}\right\|_{0} .
$$

Не ограничивая обшности будем считать, что $\left\|U_{\varepsilon_{k}}\right\|_{0}=1$. Тогда из (2.15) и (2.16) следует, что при $k \rightarrow+\infty$

$$
\begin{aligned}
\left\|U_{\varepsilon_{k}}\right\|_{1} & \leqslant 2 \mathcal{C} \\
\left\|f_{k}\right\|_{0} & <\frac{2 \mathcal{C}}{k} .
\end{aligned}
$$

Поскольку $K$ - компакт и из ограниченности множества в $H^{1}$ следует слабая компактность в $H^{1}$, то существует подпоследовательность индексов $\{k\}$, а также существуют $\lambda^{*}$ и $U^{*}$ такие, что на этой подпоследовательности при $k \rightarrow+\infty$

$$
\begin{gathered}
\lambda_{k} \rightarrow \lambda^{*} \in K, \\
U_{\varepsilon_{k}} \rightarrow U^{*} \text { слабо в } H^{1}(\Omega) \text { при } \varepsilon_{k} \rightarrow 0, U^{*} \not \equiv 0 .
\end{gathered}
$$

Пусть $v \in H^{1}\left(\Omega, \Gamma_{4}\right)$. Очевидно, что $v \in H^{1}\left(\Omega, \Gamma_{\varepsilon}\right)$. Подставим в интегральное тождество (2.12) $U=U_{\varepsilon_{k}}, \lambda=\lambda_{k}, f=f_{k}$ и упомянутое $v$. Имеем

$$
\int_{\Omega} \nabla U_{\varepsilon_{k}} \nabla \bar{v} d x-\lambda_{k} \int_{\Omega \backslash B_{\varepsilon_{k}}} U_{\varepsilon_{k}} \bar{v} d x-\lambda_{k}\left(a \varepsilon_{k}\right)^{-m} \int_{B_{\varepsilon_{k}}} U_{\varepsilon_{k}} \bar{v} d x=\int_{\Omega} f_{k} \bar{v} d x .
$$

Покажем, что третий интеграл в левой части стремится к нулю. По лемме 2.1 имеем

$$
\begin{aligned}
& \left(a \varepsilon_{k}\right)^{-m}\left|\int_{B_{\varepsilon_{k}}} U_{\varepsilon_{k}} \bar{v} d x\right| \\
& \quad \leqslant \sqrt{\left(a \varepsilon_{k}\right)^{-m} \int_{B_{\varepsilon_{k}}}\left|U_{\varepsilon_{k}}\right|^{2} d x} \sqrt{\left(a \varepsilon_{k}\right)^{-m} \int_{B_{\varepsilon_{k}}}|v|^{2} d x}
\end{aligned}
$$




$$
\begin{aligned}
& \leqslant C \sqrt{\left(a \varepsilon_{k}\right)^{2-m} \int_{B_{\varepsilon_{k}}}\left|\nabla U_{\varepsilon_{k}}\right|^{2} d x} \sqrt{\left(a \varepsilon_{k}\right)^{2-m} \int_{B_{\varepsilon_{k}}}|\nabla v|^{2} d x} \\
& \leqslant C_{2}\left(a \varepsilon_{k}\right)^{2-m},
\end{aligned}
$$

и интеграл стремится к нулю при $\varepsilon_{k} \rightarrow 0$. Таким образом, переходя к пределу в интегральном тождестве (2.12) при $\varepsilon_{k} \rightarrow 0$, используя (2.18)-(2.20), получаем новое интегральное тождество

$$
\int_{\Omega} \nabla U^{*} \nabla \bar{v} d x=\lambda^{*} \int_{\Omega} U^{*} \bar{v} d x .
$$

Из леммы 2.2 следует, что $U^{*} \in H^{1}\left(\Omega, \Gamma_{4}\right)$. Поскольку $U^{*} \not \equiv 0$, а $v$-произвольная функция из $H^{1}\left(\Omega, \Gamma_{4}\right)$, то $\lambda^{*}$ - собственное значение предельной задачи (1.3). $\mathrm{C}$ другой стороны, $\lambda^{*} \in K$. Однако мы предполагали, что $K$ не содержит собственных значений предельной задачи (1.3). Из полученного противоречия следует оценка (2.3).

2) Пусть $\lambda \in K$ - произвольное фиксированное число, а $\left\{\varepsilon_{k}\right\}$ - произвольная последовательность, сходящаяся к нулю при $k \rightarrow+\infty$. Из (2.3), леммы 2.2 , слабой компактности ограниченного множества в $H^{1}(\Omega)$, компактности вложения $H^{1}(\Omega)$ в $L_{2}(\Omega)$ и ограниченности оператора сужения $H^{1}(\Omega)$ в $L_{2}(\partial \Omega)$ следует, что существует такая подпоследовательность индексов $\{k\}$, что

$$
U_{\varepsilon_{k}} \rightarrow U^{*} \text { слабо в } H^{1}(\Omega) \text { при } \varepsilon_{k} \rightarrow 0, U^{*} \in H^{1}\left(\Omega, \Gamma_{4}\right) .
$$

Переходя к пределу в интегральном тождестве (2.13), в силу вьшесказанного, аналогично доказательству утверждения 1 ), получаем тождество

$$
\int_{\Omega} \nabla U^{*} \nabla \bar{v} d x=\lambda \int_{\Omega} U^{*} \bar{v} d x+\int_{\Omega} f \bar{v} d x,
$$

которое совпадает с интегральным тождеством задачи (2.2). Из единственности решения предельной задачи $(2.2)$ получаем, что $U^{*}=U_{0}$. Подставляя в интегральное тождество (2.13) $v=U_{\varepsilon_{k}}$ и учитывая сходимость функции в $L_{2}(\Omega)$ и лемму 2.1, получаем

$$
\left\|U_{\varepsilon_{k}}\right\|_{1}-\left\|U_{0}\right\|_{1} \rightarrow 0
$$

Из (2.23) и слабой сходимости $U_{\varepsilon_{k}}$ к $U_{0}$ в $H^{1}(\Omega)$ следует сильная сходимость в этом пространстве. Отсюда в силу произвольности $\left\{\varepsilon_{k}\right\}$ получаем справедливость сходимости (2.4).

ДОКАЗАТЕЛЬСТво ТЕОРЕМЫ 2.2. Обозначим через $S(r, z)$ открытый круг на комплексной плоскости радиуса $r$ с центром в точке $z$.

1) Пусть $\lambda_{0}$ - произвольное собственное значение предельной задачи. Поскольку собственные значения не имеют конечных точек накопления (см., например, [59]), то сушествует $R>0$ такое, что $\overline{S\left(r, \lambda_{0}\right)}$ не содержит собственных значений предельной задачи, отличных от $\lambda_{0}$, при любых $r \leqslant R$. Поэтому в силу теоремы $\left.2.1,1\right)$ 
окружность $\partial S\left(r, \lambda_{0}\right)$ не содержит собственных значений задачи (1.1). Из теоремы 2.1 и теоремы Лебега следует, что для решения задачи $(2.1)$ при $\varepsilon \rightarrow 0$ имеет место сходимость в $L_{2}(\Omega)$ при любой правой части $f \in L_{2}(\Omega)$ :

$$
\int_{\partial S\left(r, \lambda_{0}\right)} U_{\varepsilon}(x, \lambda) d \lambda \rightarrow \int_{\partial S\left(r, \lambda_{0}\right)} U_{0}(x, \lambda) d \lambda, \quad \varepsilon \rightarrow 0 .
$$

Известно, что резольвенты краевых задач (2.1) и (2.2) имеют полюсы только первого порядка, являюшиеся собственными значениями этих задач, а вычеты в них суть проекторы в $L_{2}(\Omega)$ на подпространства, образованные соответствуюшими собственные функциями. И наоборот, любое собственное значение является полюсом резольвенты первого порядка. Пусть $f$ - собственная функция $u_{0}$ оператора краевой задачи (1.3). Тогда правая часть (2.24) не равна нулю, следовательно, при достаточно малых $\varepsilon$ не равна нулю и левая часть $(2.24)$. Поэтому в $S\left(r, \lambda_{0}\right)$ существует собственное значение $\lambda_{\varepsilon}$ задачи (1.1), которое в силу произвольности в выборе $r<R$ сходится к $\lambda_{0}$ при $\varepsilon \rightarrow 0$.

2) Легко видеть, что при $\varepsilon>0$ в силу регулярной теории возмущений [62] кратность собственных значений не зависит от $\varepsilon$. Обозначим совокупную кратность собственных значений задачи $(2.1)$, сходящихся к $\lambda_{0}$ по некоторой последовательности $\varepsilon_{k} \rightarrow 0$, через $\widehat{N}$. Допустим, что $\widehat{N}$ не равно $N-$ кратности собственного значения $\lambda_{0}$. Тогда при $\varepsilon=\varepsilon_{k}$ существует $\widehat{N}$ собственных функций $u_{\varepsilon}^{j}, j=1, \ldots, \widehat{N}$, соответствующих собственным значениям, сходящимся к $\lambda_{0}$ (не ограничивая общности, будем считать, что собственные функции ортонормированы в $L_{2}(\Omega)$ ). Повторяя доказательство утверждения 2 ) теоремы 2.1 , покажем, что существует подпоследовательность $\left\{\varepsilon_{k}\right\}$ (обозначаемая так же), сходящаяся к нулю при $k \rightarrow+\infty$ и такая, что

$$
\left\|u_{\varepsilon_{k}}^{j}-u_{0}^{j}\right\|_{1} \rightarrow 0, \quad \varepsilon_{k} \rightarrow 0,
$$

где $u_{0}^{j}$ - ортонормированные в $L_{2}(\Omega)$ собственные функции предельной задачи, соответствуюшие $\lambda_{0}$. Поскольку кратность $\lambda_{0}$ равна $N$, то необходимо $\widehat{N} \leqslant N$. Допустим, что $\widehat{N}<N$. Тогда существует собственная функция $u_{0}$ предельной задачи, ортогональная $u_{0}^{j}$ при $j \leqslant \widehat{N}$. Следовательно,

$$
\sum_{j=1}^{\widehat{N}} \int_{\Omega} u_{0}(x) u_{\varepsilon}^{j}(x) d x \rightarrow 0 .
$$

Положим

$$
f(x, \varepsilon)=u_{0}(x)-\left(\sum_{j=1}^{\widehat{N}} \int_{\Omega} u_{0}(x) u_{\varepsilon}^{j}(x) d x\right) u_{\varepsilon}^{j}(x), \quad x \in \Omega .
$$

По определению имеем

$$
\sum_{j=1}^{\widehat{N}} \int_{\Omega} f(x, \varepsilon) u_{\varepsilon}^{j}(x) d x=0 .
$$


Следовательно, из (2.26) и (2.25) вытекает, что

$$
f(x, \varepsilon) \rightarrow f_{0}(x)=u_{0}(x)
$$

в $L_{2}(\Omega)$ при $\varepsilon \rightarrow 0$. Учитьвая неравенство (2.3) и сходимость $(2.4)$, легко показать, что сходимость (2.4) имеет место и для произвольных $f(x, \varepsilon)$, сходящихся в $L_{2}(\Omega)$. Следовательно, справедлива сходимость (2.24), причем интеграл в правой части не равен нулю в силу (2.28). С другой стороны, в силу (2.27) левая часть (2.24) равна нулю. Из полученного противоречия следует, что $\widehat{N}=N$. Теорема доказана.

ДОКАЗАТЕЛЬСТВО ТЕОРЕМЫ 2.3. Из утверждения 2) теоремы 2.2 следует, что при $\lambda$, близких к $\lambda_{0}$, для решения краевой задачи (2.1) справедливо представление

$$
U_{\varepsilon}(x, \lambda)=\sum_{j=1}^{N} \frac{u_{\varepsilon}^{j}(x)}{\lambda_{\varepsilon}^{j}-\lambda} \int_{\Omega} u_{\varepsilon}^{j} f d x+\widetilde{U}_{\varepsilon}(x, \lambda), \quad\left(\widetilde{U}_{\varepsilon}, u_{\varepsilon}^{j}\right)_{0}=0
$$

где $\widetilde{U}_{\varepsilon}$ - голоморфная по $\lambda \in \overline{S\left(r, \lambda_{0}\right)}$ функция для достаточно малых $r$, ортогональная всем $u_{\varepsilon}^{j}$ в $L_{2}(\Omega)$. Из $(2.29)$ и утверждения 1$)$ теоремы 2.1 следует справедливость оценки

$$
\left\|\widetilde{U}_{\varepsilon}\right\|_{1} \leqslant C\|f\|_{0}
$$

при $\lambda \in \partial S\left(r, \lambda_{0}\right)$. Следовательно, эта оценка верна и для $\lambda \in \overline{S\left(r, \lambda_{0}\right)}$. Из $(2.29)$ и (2.30) вытекает справедливость утверждения 1) доказываемой теоремы.

Поскольку $\widetilde{U}_{\varepsilon}$ ортогонально $u_{\varepsilon}^{j}$ в $L_{2}(\Omega)$ для фиксированного $j=1, \ldots N$, то справедливость утверждения 2) следует также из (2.29) и (2.30). Теорема доказана.

Теорема 2.3 будет в дальнейшем использована для оценки невязки и обоснования формального асимптотического разложения.

\section{§3. Простые собственные значения}

3.1. Формальный асимптотический анализ. Построение ведущих членов. Для построения асимптотик мы воспользуемся методом согласования асимптотических разложений (см. [63]-[66], а также [68]-[70]).

Предполагается, что в спектральной задаче (1.3) $\lambda_{0}$ - простое и для определенности всюду далее в этом параграфе считаем, что функция $u_{0}(x)$ нормирована в $L_{2}(\Omega)$. Естественно искать разложение решения в форме

$$
u_{\varepsilon}(x)=u_{0}(x)+\ldots,
$$

а также разложение собственных значений в виде

$$
\lambda_{\varepsilon}=\lambda_{0}+\ldots
$$

Функция $u_{0}(x)$ не удовлетворяет ни уравнению, ни граничным условиям исходной задачи в окрестности $\Gamma_{4}$, поэтому ее надо “подправить" в этой окрестности. 
Следуя терминологии метода согласования, разложение (3.1) назовем внешним, а разложение в малой окрестности $\Gamma_{4}$ назовем внутренним.

Отметим, что внешним разложением мы будем пользоваться во всей области, за исключением малой окрестности участка границы $\Gamma_{4}$, в котором будем строить другой ряд (внутреннее разложение). Поэтому нас интересуют в первую очередь не сами граничные условия на функции внешнего разложения (они введены для корректности задач), а асимптотическое поведение членов ряда при $x_{2} \rightarrow 0$.

Собственную функцию $u_{0}(x)$ разложим в ряд Тейлора по переменной $x_{2}$ при $x_{2} \rightarrow 0$. В силу краевой задачи (1.3) (уравнения и граничных условий на $\Gamma_{2}$, $\left.\Gamma_{3}, \Gamma_{4}\right)$ имеем

$$
\begin{gathered}
u_{0}(x)=\alpha_{0}\left(x_{1}\right) x_{2}+O\left(x_{2}^{3}\right), \\
\alpha_{0}^{\prime}\left( \pm \frac{\pi}{2}\right)=0
\end{gathered}
$$

где

$$
\alpha_{0}\left(x_{1}\right)=\left.\frac{\partial u_{0}}{\partial x_{2}}\right|_{x_{2}=0} .
$$

Сделаем замену $\xi_{2}=\frac{x_{2}}{\varepsilon}$. Получаем в силу $(3.3)$

$$
u_{0}\left(x_{1}, \varepsilon \xi_{2}\right)=\varepsilon \alpha_{0}\left(x_{1}\right) \xi_{2}+O\left(\varepsilon^{3} \xi_{2}^{3}\right)
$$

Главный член внутреннего разложения должен иметь "правильные" граничные условия (как в задаче (1.1)) и такую же асимптотику, как в (3.5), но на бесконечности (при $\left.\xi_{2} \rightarrow+\infty\right)$ (в этом и заключается метод согласования разложений $[66]$ ). Следовательно, внутреннее разложение должно иметь структуру

$$
u_{\varepsilon}(x)=\varepsilon v_{1}\left(\xi ; x_{1}\right)+\ldots,
$$

где $v_{1}\left(\xi ; x_{1}\right) \sim \alpha_{0}\left(x_{1}\right) \xi_{2}$ при $\xi_{2} \rightarrow+\infty$, а $\xi=\frac{x}{\varepsilon}$. Здесь $x_{1}$ играет роль "медленного времени". В переменных $\left(\xi ; x_{1}\right)$ уравнение задачи $(1.1)$ имеет вид

$$
-\varepsilon^{-2} \Delta_{\xi} u_{\varepsilon}-2 \varepsilon^{-1} \frac{\partial^{2} u_{\varepsilon}}{\partial x_{1} \partial \xi_{1}}-\frac{\partial^{2} u_{\varepsilon}}{\partial x_{1}^{2}}=\lambda_{\varepsilon} \rho_{\varepsilon} u_{\varepsilon}
$$

с граничными условиями на $\Gamma_{2}$

$$
\frac{\partial u_{\varepsilon}}{\partial \nu}=-\varepsilon^{-1} \frac{\partial u_{\varepsilon}}{\partial \xi_{1}}-\frac{\partial u_{\varepsilon}}{\partial x_{1}}=0
$$

и на $\Gamma_{3}$

$$
\frac{\partial u_{\varepsilon}}{\partial \nu}=\varepsilon^{-1} \frac{\partial u_{\varepsilon}}{\partial \xi_{1}}+\frac{\partial u_{\varepsilon}}{\partial x_{1}}=0
$$


ЗАмЕчАниЕ 3.1. Далее мы будем строить члены внутреннего разложения (3.6) в виде $\pi$-периодических по $\xi_{1}$ функций (для каждого фиксированного значения $x_{1}$ ).

Подставляем (3.2), (3.6) в (1.1) и, учитывая (3.7)-(3.9), собираем члены с минимальными степенями $\varepsilon$ (в уравнении с $\varepsilon^{-1}$, в граничных условиях с $\varepsilon^{0}$ ). С учетом замечания 3.1 получаем задачу

$$
\begin{gathered}
\Delta_{\xi} v_{1}=0 \text { в } \Pi, \\
v_{1}=0 \text { на } \gamma, \quad \frac{\partial v_{1}}{\partial \xi_{2}}=0 \text { на } \Gamma, \\
\frac{\partial v_{1}}{\partial \xi_{1}}\left(\xi ; \pm \frac{\pi}{2}\right)=0 \text { при } \xi_{1}= \pm \frac{\pi}{2}, \\
v_{1} \sim \alpha_{0} \xi_{2} \text { при } \xi_{2} \rightarrow+\infty .
\end{gathered}
$$

Рассмотрим вспомогательную задачу

$$
\begin{gathered}
\Delta_{\xi} X=0 \text { в } \Pi, \\
X=0 \text { на } \gamma, \quad \frac{\partial X}{\partial \xi_{2}}=0 \text { на } \Gamma, \\
\frac{\partial X}{\partial \xi_{1}}=0 \text { при } \xi_{1}= \pm \frac{\pi}{2}, \\
X \sim \xi_{2} \text { при } \xi_{2} \rightarrow+\infty .
\end{gathered}
$$

Решение задачи (3.11) сушествует (см. [68], а также [69] и [70]). Его можно выписать явно:

$$
X(\xi)=\operatorname{Re} \ln \left(\sin z+\sqrt{\sin ^{2} z-\sin ^{2} a}\right)-\ln \sin a,
$$

где $z=\xi_{1}+i \xi_{2}$, причем $X(\xi)$ продолжается периодически по $\xi_{1}$. Поэтому ясно, что

$$
v_{1}\left(\xi ; x_{1}\right)=\alpha_{0}\left(x_{1}\right) X(\xi)
$$

является решением задачи (3.10). Это $\pi$-периодическая по $\xi_{1}$ и четная по $\xi_{1}$ функция. Асимптотика такой функции на бесконечности при $\xi_{2} \rightarrow+\infty$ в силу (3.12) и (3.13) выглядит следующим образом:

$$
v_{1}\left(\xi ; x_{1}\right)=\alpha_{0}\left(x_{1}\right)\left[\left(\xi_{2}-\ln \sin a\right)+O\left(e^{-2 \xi_{2}}\right)\right]
$$

Отметим, что благодаря (3.4) выполняется

$$
\frac{\partial v_{1}}{\partial x_{1}}\left(\xi ; x_{1}\right)=0 \text { при } x_{1}= \pm \frac{\pi}{2},
$$

и, следовательно, в силу (3.8)- (3.10) и (3.15) имеем

$$
\frac{\partial v_{1}}{\partial \nu}\left(\frac{x}{\varepsilon} ; x_{1}\right)=0 \text { на } \Gamma_{2} \cup \Gamma_{3}
$$


Теперь видно, что, устранив невязку в граничных условиях для функции $u_{0}(x)$, мы построили $v_{1}\left(\xi ; x_{1}\right)$, но при этом оказалось, что поведение на бесконечности функции $v_{1}\left(\xi ; x_{1}\right)$ не совсем такое, которое нам было необходимо. Появилась новая невязка, но другого (меньшего) порядка. Появилась ограниченная функция от $x_{1}$, умноженная на первую степень $\varepsilon$. Эту невязку приходится устранять введением следуюшего члена во внешнем разложении.

Переписьвая асимптотику $\varepsilon v_{1}$ в бесконечности во внешних переменных с учетом (3.14), заключаем, что внешнее разложение должно иметь вид

$$
u_{\varepsilon}(x)=u_{0}(x)+\varepsilon u_{1}(x)+\ldots,
$$

где

$$
u_{1}\left(x_{1}, x_{2}\right) \sim-\alpha_{0}\left(x_{1}\right) \ln \sin a \text { при } x_{2} \rightarrow 0 .
$$

Мы будем искать гладкую функцию $u_{1}$, поэтому такая асимптотика эквивалентна граничным условиям

$$
u_{1}\left(x_{1}, 0\right)=-\alpha_{0}\left(x_{1}\right) \ln \sin a, \quad x_{1} \in\left(-\frac{\pi}{2}, \frac{\pi}{2}\right) .
$$

Мы нашли краевые условия для $u_{1}(x)$ на части гранищы $\Gamma_{4}$. Поскольку уточненное внешнее разложение имеет вид (3.17), то, естественно, разложение собственного значения в уточненном виде будет иметь следующий член порядка $\varepsilon$, т. е.

$$
\lambda_{\varepsilon}=\lambda_{0}+\varepsilon \lambda_{1}+\ldots
$$

где константу $\lambda_{1}$ мы вычислим ниже.

Подставляя ряд (3.17) и разложение (3.19) для $\lambda_{\varepsilon}$ в задачу (1.1), приравнивая члены при $\varepsilon^{1}$ и учитывая граничное условие $(3.18)$, получаем краевую задачу для $u_{1}$ :

$$
\begin{gathered}
-\Delta u_{1}=\lambda_{0} u_{1}+\lambda_{1} u_{0} \text { в } \Omega, \\
u_{1}=-\alpha_{0} \ln \sin a \text { на } \Gamma_{4}, \\
\frac{\partial u_{1}}{\partial \nu}=0 \text { на } \Gamma_{1} \cup \Gamma_{2} \cup \Gamma_{3} .
\end{gathered}
$$

Записываем условие разрешимости задачи (3.20). Домножим уравнение задачи на $u_{0}$ и проинтегрируем по области $\Omega$, учитьвая нормировку $u_{0}$. Имеем

$$
-\int_{\Omega} \Delta u_{1}(x) u_{0}(x) d x=\lambda_{0} \int_{\Omega} u_{1}(x) u_{0}(x) d x+\lambda_{1} \int_{\Omega} u_{0}^{2}(x) d x .
$$

Применяя дважды формулу Грина к левой части равенства (3.21), выводим

$$
\begin{aligned}
-\int_{\Omega} \Delta u_{1} u_{0} d x & =\int_{\Omega}\left(\nabla u_{1}, \nabla u_{0}\right) d x-\int_{\partial \Omega} \frac{\partial u_{1}}{\partial \nu} u_{0} d s \\
& =-\int_{\Omega} u_{1} \Delta u_{0} d x-\int_{\partial \Omega} \frac{\partial u_{1}}{\partial \nu} u_{0} d s+\int_{\partial \Omega} \frac{\partial u_{0}}{\partial \nu} u_{1} d s \\
& =\lambda_{0} \int_{\Omega} u_{1} u_{0} d x+\ln \sin a \int_{-\frac{\pi}{2}}^{\frac{\pi}{2}} \alpha_{0}^{2} d x_{1}
\end{aligned}
$$


Здесь мы воспользовались тем, что на $\Gamma_{4} \frac{\partial u_{0}}{\partial \nu}=-\frac{\partial u_{0}}{\partial x_{2}}=-\alpha_{0}$. Подставляя (3.22) в (3.21), получаем

$$
\lambda_{1}=\ln \sin a \int_{-\frac{\pi}{2}}^{\frac{\pi}{2}} \alpha_{0}^{2}\left(x_{1}\right) d x_{1},
$$

или

$$
\lambda_{1}=\ln \sin a \int_{\Gamma_{4}}\left(\frac{\partial u_{0}}{\partial \nu}\right)^{2} d s .
$$

Для однозначной разрешимости задачи (3.20) будем считать, что

$$
\int_{\Omega} u_{0}(x) u_{1}(x) d x=0 .
$$

Продолжаем процесс. Разложим функцию $u_{1}(x)$ в ряд Тейлора в нуле. Легко видеть, что $u_{1}(x)$ - гладкая и ряд Тейлора имеет вид

$$
u_{1}(x)=u_{1}\left(x_{1}, 0\right)+\frac{\partial u_{1}}{\partial x_{2}}\left(x_{1}, 0\right) x_{2}+\ldots
$$

или

$$
u_{1}(x)=-\alpha_{0}\left(x_{1}\right) \ln \sin a+\alpha_{1}\left(x_{1}\right) x_{2}+\ldots,
$$

где

$$
\alpha_{1}\left(x_{1}\right)=\frac{\partial u_{1}}{\partial x_{2}}\left(x_{1}, 0\right) .
$$

Отметим, что благодаря гладкости функции $u_{1}$ и однородности условия Неймана на $\Gamma_{2}$ и $\Gamma_{3}$ для $u_{1}$ мы получаем

$$
\alpha_{1}^{\prime}\left( \pm \frac{\pi}{2}\right)=0
$$

Перепишем (3.24) в координатах $\left(x_{1}, \xi_{2}\right)$. Соответственно, получаем

$$
u_{1}\left(x_{1}, \varepsilon \xi_{2}\right)=-\alpha_{0}\left(x_{1}\right) \ln \sin a+\varepsilon \alpha_{1}\left(x_{1}\right) \xi_{2}+\ldots .
$$

Строя внешнее разложение, мы получили новую невязку в асимптотике при $x_{2} \rightarrow 0$ вида $\varepsilon^{2} \alpha_{1}\left(x_{1}\right)$ (см. $\left.(3.17),(3.24)\right)$. Следуюший член внутреннего ряда (член порядка $\varepsilon^{2}$ ) должен опять иметь "правильные" граничные условия и асимптотику на бесконечности, компенсирующую эту невязку. А именно, переписывая асимптотику $u_{0}+\varepsilon u_{1}$ при $x_{2} \rightarrow 0$ в переменных $\xi$, получаем, с учетом (3.3) и (3.24), внутреннее разложение в форме

$$
u_{\varepsilon}(x)=\varepsilon v_{1}\left(\xi ; x_{1}\right)+\varepsilon^{2} v_{2}\left(\xi ; x_{1}\right)+\ldots,
$$

где

$$
v_{2}\left(\xi ; x_{1}\right) \sim \alpha_{1}\left(x_{1}\right) \xi_{2} \text { при } \xi_{2} \rightarrow \infty .
$$

До того как мы будем строить член порядка $\varepsilon^{2}$ во внутреннем разложении, обратим внимание на то, что при $1<m<2$ в уравнении задачи $(1.1)$, переписанном 
во внутренних переменных, появляется как минимум еще один некомпенсированный член. Действительно,

$$
-\varepsilon^{-2} \Delta_{\xi} v_{1}-\ldots=\left(\lambda_{0}+\varepsilon \lambda_{1}+\ldots\right) \rho_{\varepsilon}\left(\varepsilon v_{1}+\ldots\right) .
$$

Подставляя $\rho_{\varepsilon}(x)$ в это уравнение, убеждаемся, что в правой части равенства при $\varepsilon^{1-m}$ есть слагаемое $a^{-m} \lambda_{0} v_{1}$, которое компенсируется введением члена $\varepsilon^{3-m} v_{3-m}$ во внутреннее разложение $u_{\varepsilon}(x)$. Видно, что при $1<m<2$ это слагаемое по порядку больше $\varepsilon^{1}$ и меньше $\varepsilon^{2}$, т. е.

$$
u_{\varepsilon}(x)=\varepsilon v_{1}\left(\xi ; x_{1}\right)+\varepsilon^{3-m} v_{3-m}\left(\xi ; x_{1}\right)+\ldots
$$

Рассмотрим случай $1<m<2$. Подставляя ряд (3.28) в задачу (1.1) и приравнивая соответствуюшие члены, получаем в силу замечания 3.1 еще одну задачу:

$$
\begin{aligned}
& -\Delta_{\xi} v_{3-m}=\left\{\begin{array}{lll}
0 & \text { в } & \Pi \backslash B, \\
a^{-m} \lambda_{0} v_{1} & \text { в } & B,
\end{array}\right. \\
& v_{3-m}=0 \text { на } \gamma, \quad \frac{\partial v_{3-m}}{\partial \xi_{2}}=0 \text { на } \Gamma \text {, } \\
& \frac{\partial v_{3-m}}{\partial \xi_{1}}\left(\xi ; \pm \frac{\pi}{2}\right)=0 \text { при } \xi_{1}= \pm \frac{\pi}{2} .
\end{aligned}
$$

Рассмотрим вспомогательную задачу

$$
\begin{gathered}
-\Delta_{\xi} X_{3-m}= \begin{cases}0 & \text { в } \Pi \backslash B, \\
a^{-m} \lambda_{0} X & \text { в } B,\end{cases} \\
X_{3-m}=0 \text { на } \gamma, \quad \frac{\partial X_{3-m}}{\partial \xi_{2}}=0 \text { на } \Gamma, \\
\frac{\partial X_{3-m}}{\partial \xi_{1}}=0 \text { при } \xi_{1}= \pm \frac{\pi}{2} .
\end{gathered}
$$

Отображая полуполосу П на единичный круг, докажем существование решения задачи (3.30) с асимптотикой

$$
X_{3-m}=\xi_{2}+c_{3-m}+O\left(e^{-2 \xi_{2}}\right) \text { при } \xi_{2} \rightarrow+\infty \text {. }
$$

Благодаря четности функции $X(\xi)$ по $\xi_{1}$ и симметричности ячейки П решение $X_{3-m}$ будет четной функцией, поэтому оно может быть периодически продолжено по $\xi_{1}$.

Очевидно, что функция

$$
v_{3-m}\left(\xi ; x_{1}\right)=\alpha_{0}\left(x_{1}\right) X_{3-m}(\xi)
$$

удовлетворяет задаче (3.29) и имеет асимптотику

$$
v_{3-m}\left(\xi ; x_{1}\right)=\alpha_{0}\left(x_{1}\right)\left(\left(\xi_{2}+c_{3-m}\right)+O\left(e^{-2 \xi_{2}}\right)\right) \text { при } \xi_{2} \rightarrow+\infty .
$$


Константу $c_{3}-m$ легко посчитать. Действительно, домножим уравнение задачи (3.30) на функцию $X(\xi)$, определенную в (3.12), и проинтегрируем по области $\Pi_{R}=\left\{\xi \in \Pi, \xi_{2}<R\right\}$. После этого дважды применим формулу Грина и перейдем к пределу при $R \rightarrow \infty$. Получим

$$
a^{-m} \lambda_{0} \int_{B} X^{2}(\xi) d \xi=\pi c_{3-m} .
$$

Отметим, что благодаря (3.4) и (3.32) выполняется

$$
\frac{\partial v_{3-m}}{\partial x_{1}}\left(\xi ; x_{1}\right)=0 \text { при } x_{1}= \pm \frac{\pi}{2}
$$

и, следовательно, в силу (3.8), (3.9), (3.29) и (3.35) имеем

$$
\frac{\partial v_{3-m}}{\partial \nu}\left(\frac{x}{\varepsilon} ; x_{1}\right)=0 \text { на } \Gamma_{2} \cup \Gamma_{3} .
$$

Появление функции $c_{3-m} \alpha_{0}\left(x_{1}\right)$, умноженной на $\varepsilon^{3-m}$, породило невязку во внешнем разложении. Эту невязку мы устраняем на следуюшем шаге, вводя во внешнее разложение член порядка $\varepsilon^{3-m}$.

Переписьвая асимптотику $\varepsilon v_{1}+\varepsilon^{3-m} v_{3-m}$ во внешних переменных с учетом (3.14) и (3.33), заключаем, что внешнее разложение должно иметь вид

$$
u_{\varepsilon}(x)=u_{0}(x)+\varepsilon u_{1}(x)+\varepsilon^{3-m} u_{3-m}(x)+\ldots,
$$

где

$$
u_{3-m}(x) \sim c_{3-m} \alpha_{0}\left(x_{1}\right) \text { при } x_{2} \rightarrow 0 .
$$

При этом граничные условия на $\Gamma_{4}$ должны быть

$$
u_{3-m}\left(x_{1}, 0\right)=c_{3-m} \alpha_{0}\left(x_{1}\right)
$$

т. е. мы нашли краевые условия для $u_{3}-m(x)$ на части границы $\Gamma_{4}$.

Теперь найдем уравнение, которому удовлетворяет $u_{3-m}(x)$. Подставляя ряд (3.37) в задачу (1.1) и проводя анализ, аналогичный сделанному выше для $u_{1}$, заключаем, что, во-первых, добавился член $\varepsilon^{3-m} \lambda_{3-m}$ в разложение $\lambda_{\varepsilon}$, а во-вторых, уравнение принимает вид

$$
\begin{aligned}
& -\Delta u_{0}-\varepsilon \Delta u_{1}-\varepsilon^{3-m} \Delta u_{3-m}-\ldots \\
& \quad=\left(\lambda_{0}+\varepsilon \lambda_{1}+\varepsilon^{3-m} \lambda_{3-m}+\ldots\right) \rho_{\varepsilon}\left(u_{0}+\varepsilon u_{1}+\varepsilon^{3-m} u_{3-m}+\ldots\right) .
\end{aligned}
$$

Приравнивая члены при $\varepsilon^{3-m}$ и учитьвая граничное условие $(3.38)$, получаем задачу для $u_{3-m}$ :

$$
\begin{gathered}
-\Delta u_{3-m}=\lambda_{0} u_{3-m}+\lambda_{3-m} u_{0} \text { в } \Omega, \\
u_{3-m}=c_{3-m} \alpha_{0} \text { на } \Gamma_{4}, \\
\frac{\partial u_{3-m}}{\partial \nu}=0 \text { на } \Gamma_{1} \cup \Gamma_{2} \cup \Gamma_{3} .
\end{gathered}
$$


Как уже отмечалось, это задача "на спектре". Записываем условие разрешимости. Домножим уравнение на $u_{0}(x)$ и проинтегрируем по области $\Omega$. После этого дважды применяем формулу Грина к левой части равенства. Воспользовавшись тем, что на $\Gamma_{4}$

$$
\frac{\partial u_{0}}{\partial \nu}=-\frac{\partial u_{0}}{\partial x_{2}}=-\alpha_{0}
$$

а также тем, что $\left\|u_{0}\right\|_{L_{2}(\Omega)}=1$, получаем

$$
\lambda_{3-m}=-\int_{-\frac{\pi}{2}}^{\frac{\pi}{2}} c_{3-m} \alpha_{0}^{2} d x_{1} .
$$

Наконец, учитьвая вид константы $c_{3-m}(3.34)$, перепишем полученное выражение. Имеем

$$
\lambda_{3-m}=-\frac{a^{-m} \lambda_{0}}{\pi} \int_{\Gamma_{4}}\left(\frac{\partial u_{0}}{\partial \nu}\right)^{2} d s \int_{B} X^{2}(\xi) d \xi .
$$

Для однозначной разрешимости задачи (3.39) будем считать, что

$$
\int_{\Omega} u_{0}(x) u_{3-m}(x) d x=0 .
$$

ЗАМЕчАниЕ 3.2. При $m$, близких к 2 , может возникнуть еще много членов с не целыми степенями $\varepsilon$ до появления члена порядка $\varepsilon^{2}$. Действительно, например, член с номером $5-2 m$, который появляется естественным образом из-за вклада $v_{3}-m$ в правую часть уравнения, при $m>\frac{3}{2}$ становится старшим по отношению к члену порядка $\varepsilon^{2}$.

Теперь рассмотрим случай $0<m<1$.

Рассмотрим внутреннее разложение (3.26). В координатах $\xi$ наше уравнение, как было отмечено вьше, имеет вид (3.7). Подставим внутреннее разложение в задачу и приравняем члены при соответствуюших степенях $\varepsilon$. Получим

$$
\begin{gathered}
-\Delta_{\xi} v_{2}=2 \frac{\partial^{2} v_{1}}{\partial x_{1} \partial \xi_{1}} \text { в } \Pi, \\
v_{2}=0 \text { на } \gamma, \quad \frac{\partial v_{2}}{\partial \xi_{2}}=0 \text { на } Г, \\
\frac{\partial v_{2}}{\partial \xi_{1}}\left(\xi ; \pm \frac{\pi}{2}\right)=0 \text { при } \xi_{1}= \pm \frac{\pi}{2},
\end{gathered}
$$

при этом асимптотика на бесконечности имеет вид

$$
v_{2}\left(\xi ; x_{1}\right) \sim \alpha_{1}\left(x_{1}\right) \xi_{2} \text { при } \xi_{2} \rightarrow+\infty .
$$

Рассмотрим вспомогательную задачу:

$$
\begin{gathered}
\Delta_{\xi} \widetilde{X}=\frac{\partial X}{\partial \xi_{1}} \text { в } \Pi, \\
\widetilde{X}=0 \text { на } \gamma, \quad \frac{\partial \widetilde{X}}{\partial \xi_{2}}=0 \quad \text { на } \Gamma, \\
\widetilde{X}=0 \text { при } \xi_{1}= \pm \frac{\pi}{2}
\end{gathered}
$$


с асимптотикой на бесконечности

$$
\widetilde{X}=O\left(\xi_{2} e^{-2 \xi_{2}}\right) \text { при } \xi_{2} \rightarrow+\infty \text {. }
$$

Доказательство существования такой функции $\widetilde{X}$ может быть проведено с помошью конформного отображения полуполосы в единичный круг. В силу нечетности правой части по $\xi_{1}$ функция $\widetilde{X}$ является нечетной по $\xi_{1}$, а в силу $\pi$-периодичности правой части и граничных условий она допускает $\pi$-периодическое продолжение по $\xi_{1}$. Сохраним для продолженной функции то же обозначение.

Благодаря (3.4), (3.12), (3.25), (3.34) и (3.45) функция

$$
v_{2}\left(\xi ; x_{1}\right)=\alpha_{1}\left(x_{1}\right) X(\xi)-2 \alpha_{0}^{\prime}\left(x_{1}\right) \tilde{X}(\xi)
$$

является $\pi$-периодическим решением задачи (3.42) с асимптотикой на бесконечности

$$
v_{2}\left(\xi ; x_{1}\right) \sim \alpha_{1}\left(x_{1}\right) \xi_{2}-\alpha_{1}\left(x_{1}\right) \ln \sin a .
$$

Отметим также, что в силу $(3.25)$ и краевого условия $\widetilde{X}\left( \pm \frac{\pi}{2}, \xi_{2}\right)=0$ имеет место

$$
\frac{\partial v_{2}}{\partial x_{1}}\left( \pm \frac{\pi}{2}, \xi_{2} ; x_{1}\right)=0 \text { при } x_{1}= \pm \frac{\pi}{2},
$$

следовательно, в силу $(3.48),(3.4)$ и условия $\frac{\partial X}{\partial \xi_{1}}\left( \pm \frac{\pi}{2}, \xi_{2}\right)=0$ выполняется равенство

$$
\frac{\partial v_{2}}{\partial \nu}\left(\frac{x}{\varepsilon} ; x_{1}\right)=0 \quad \text { на } \quad \Gamma_{2} \cup \Gamma_{3}
$$

Появление функции $-\alpha_{1}\left(x_{1}\right) \ln \sin a$, умноженной на $\varepsilon^{2}$, очередной раз “испортило" внешнее разложение. Эту невязку мы устраняем на следуюшем шаге, вводя во внешнее разложение член порядка $\varepsilon^{2}$. Добавляем следующий член во внешнее разложение; с учетом переписанной асимптотики $\varepsilon v_{1}+\varepsilon^{2} v_{2}$ в бесконечности во внешних переменных и гладкости $u_{2}$ получаем

$$
u_{\varepsilon}(x)=u_{0}(x)+\varepsilon u_{1}(x)+\varepsilon^{2} u_{2}(x)+\ldots,
$$

где

$$
u_{2}\left(x_{1}, 0\right)=-\alpha_{1}\left(x_{1}\right) \ln \sin a,
$$

т. е. мы нашли краевые условия для $u_{2}(x)$ на части гранищы $\Gamma_{4}$.

Теперь найдем уравнение, которому удовлетворяет $u_{2}(x)$. Подставляя ряд (3.50) и разложение $\lambda_{\varepsilon}$ в задачу (1.1), приравнивая члены при $\varepsilon^{2}$ и проводя анализ, аналогичный сделанному выше для $u_{1}$ и $u_{3-m}$, получаем задачу для $u_{2}$ :

$$
\begin{gathered}
-\Delta u_{2}=\lambda_{0} u_{2}+\lambda_{1} u_{1}+\lambda_{2} u_{0} \text { в } \Omega, \\
u_{2}=-\alpha_{1} \ln \sin a \text { на } \Gamma_{4}, \\
\frac{\partial u_{2}}{\partial \nu}=0 \text { на } \Gamma_{1} \cup \Gamma_{2} \cup \Gamma_{3} .
\end{gathered}
$$


Записываем условие разрешимости для этой задачи. Домножим уравнение на $u_{0}(x)$ и проинтегрируем по области $\Omega$, применяя дважды формулу Грина к левой части равенства и учитывая, что $\left\|u_{0}\right\|_{L_{2}(\Omega)}=1$ и $\int_{\Omega} u_{0}(x) u_{1}(x) d x=0$. Получаем

$$
\lambda_{2}=\ln \sin a \int_{-\frac{\pi}{2}}^{\frac{\pi}{2}} \alpha_{1}\left(x_{1}\right) \alpha_{0}\left(x_{1}\right) d x_{1}=\ln \sin a \int_{\Gamma_{4}} \frac{\partial u_{0}}{\partial \nu} \frac{\partial u_{1}}{\partial \nu} d s .
$$

Для однозначной разрешимости задачи (3.50) будем считать, что

$$
\int_{\Omega} u_{0}(x) u_{2}(x) d x=1
$$

Отдельно рассмотрим случай $m=1$. Легко заметить, что в этом случае задача для $v_{3-m}$ и задача для $v_{2}$ "сливаются" в одну. Поэтому и задачи для $u_{3-m}$ и $u_{2}$ оказываются связанными. Легко проверить, что при этом задача для $v_{2}$ имеет вид

$$
\begin{gathered}
-\Delta_{\xi} v_{2}=2 \frac{\partial^{2} v_{1}}{\partial x_{1} \partial \xi_{1}}+ \begin{cases}0 & \text { в } \Pi \backslash B, \\
a^{-1} \lambda_{0} v_{1} & \text { в } B,\end{cases} \\
v_{2}=0 \text { на } \gamma, \quad \frac{\partial v_{2}}{\partial \xi_{2}}=0 \text { на } \Gamma, \\
\frac{\partial v_{2}}{\partial \xi_{1}}\left(\xi ; \pm \frac{\pi}{2}\right)=0 \text { при } \xi_{1}= \pm \frac{\pi}{2},
\end{gathered}
$$

т. е. по сути решение этой задачи равно сумме решений задач (3.29) и (3.42).

Эта функция имеет асимптотику

$$
v_{2}\left(\xi ; x_{1}\right) \sim \alpha_{1}\left(x_{1}\right)\left(\xi_{2}-\ln \sin a\right)+c_{3-m} \alpha_{0}\left(x_{1}\right) \text { при } \xi_{2} \rightarrow+\infty
$$

при этом

$$
\begin{gathered}
-\Delta u_{2}=\lambda_{0} u_{2}+\lambda_{1} u_{1}+\lambda_{2} u_{0} \text { в } \Omega, \\
u_{2}=-\alpha_{1} \ln \sin a+c_{3-m} \alpha_{0} \text { на } \Gamma_{4}, \\
\frac{\partial u_{2}}{\partial \nu}=0 \text { на } \Gamma_{1} \cup \Gamma_{2} \cup \Gamma_{3} .
\end{gathered}
$$

Опять отметим, что решение задачи (3.55) есть сумма решений задач (3.39) и (3.51).

Собственные числа вычисляются по формуле

$$
\lambda_{2}=\lambda_{2}^{\text {old }}+\lambda_{3-m},
$$

где $\lambda_{2}^{\text {old }}$ задана в $(3.52)$, а $\lambda_{3-m}$ задана в (3.41).

В случае, если $m$ близко к 2 , мы обнаруживаем появление дополнительных членов, порядки которых меньше двух. Для аккуратного обоснования построенных членов необходимо выписать полное разложение для решения $u_{\varepsilon}$ и собственного значения $\lambda_{\varepsilon}$. 
3.2. Построение полной асимптотики. Построим полное асимптотическое разложение собственных значений и собственных функций задачи (1.1) и строго обоснуем его.

Перенумеруем множество $\{1+i+(2-m) j\}_{i, j=0}^{\infty}$ для фиксированного $m$ в порядке возрастания: $1=\varsigma_{1}<\varsigma_{2}<\ldots$. Легко видеть, что $\varsigma_{2}=2$ при $0<m \leqslant 1$ и $\varsigma_{2}=3-m$ при $1<m<2$.

Пусть $0<\beta<1-$ произвольное число. В этом пункте проведем доказательство следующего утверждения.

ТЕОРема 3.1. Асимптотика собственных значений и соответствующих собственных функиий имеет вид

$$
\begin{gathered}
\lambda_{\varepsilon}=\lambda_{0}+\sum_{i=1}^{\infty} \varepsilon^{\varsigma_{i}} \lambda_{\varsigma_{i}}, \\
\mathcal{U}_{\varepsilon}(x)=u_{0}(x)+\sum_{i=1}^{\infty} \varepsilon^{\varsigma_{i}} u_{\varsigma_{i}}(x), \quad x_{2}>\varepsilon^{\beta}, \\
\mathcal{U}_{\varepsilon}(x)=\sum_{i=1}^{\infty} \varepsilon^{\varsigma_{i}} v_{\varsigma_{i}}\left(\frac{x}{\varepsilon} ; x_{1}\right), \quad 0 \leqslant x_{2}<\varepsilon^{\beta},
\end{gathered}
$$

в норме пространства Соболева $H^{1}(\Omega)$. Здесь $u_{\varsigma_{k}} \in C^{\infty}(\bar{\Omega}), \quad v_{\varsigma_{k}}\left(\xi ; x_{1}\right)-$ $\pi$-периодические по $\xi_{1}$ функиии с асимптотикой

$$
v_{\varsigma_{i}}\left(\xi ; x_{1}\right) \sim P_{\left[\varsigma_{i}\right]}\left(\xi_{2}\right) \quad \text { npu } \quad \xi_{2} \rightarrow \infty
$$

для любого фиксированного $x_{1}$, где $\left[\varsigma_{i}\right]-$ целая часть $\varsigma_{i}, P_{k}\left(\xi_{2}\right)$-многочлен степени $k$,

$$
\begin{gathered}
v_{\varsigma_{1}}\left(\xi ; x_{1}\right)=\alpha_{0}\left(x_{1}\right) X(\xi), \\
\lambda_{\varsigma_{1}}=\ln \sin a \int_{-\frac{\pi}{2}}^{\frac{\pi}{2}} \alpha_{0}^{2}\left(x_{1}\right) d x_{1}, \\
\lambda_{\varsigma_{2}}=-\frac{a^{-m} \lambda_{0}}{\pi} \int_{-\frac{\pi}{2}}^{\frac{\pi}{2}} \alpha_{0}^{2}\left(x_{1}\right) d x_{1} \int_{B} X^{2}(\xi) d \xi \quad n p u \quad 1<m<2, \\
\lambda_{\varsigma_{2}}=\ln \sin a \int_{-\frac{\pi}{2}}^{\frac{\pi}{2}} \alpha_{1}\left(x_{1}\right) \alpha_{0}\left(x_{1}\right) d x_{1} \\
-\delta_{m}^{1} \frac{a^{-m} \lambda_{0}}{\pi} \int_{-\frac{\pi}{2}}^{\frac{\pi}{2}} \alpha_{0}^{2}\left(x_{1}\right) d x_{1} \int_{B} X^{2}(\xi) d \xi \quad n p u \quad 0<m \leqslant 1,
\end{gathered}
$$

əде $X(\xi)=\operatorname{Re} \ln \left(\sin z+\sqrt{\sin ^{2} z-\sin ^{2} a}\right)-\ln \sin a, \quad z=\xi_{1}+i \xi_{2}, \quad \alpha_{0}\left(x_{1}\right)=$ $\left.\frac{\partial u_{0}}{\partial x_{2}}\right|_{x_{2}=0}, \quad \alpha_{1}\left(x_{1}\right)=\left.\frac{\partial u_{1}}{\partial x_{2}}\right|_{x_{2}=0}, \quad u_{1}-$ решение задачи $(3.20)$, ортогональное $u_{0}$ в $L_{2}(\Omega), \delta_{k}^{l}-$ символ Кронекера. 
ЗАмЕчанИЕ 3.3 . Здесь $\mathcal{U}_{\varepsilon}(x)=T_{\varepsilon} u_{\varepsilon}(x)$, константа $T_{\varepsilon}=1+o(1)$ при $\varepsilon \rightarrow 0$.

Подставляя ряд (3.57) и разложение $(3.56)$ для $\lambda_{\varepsilon}$ в задачу $(1.1)$, приравнивая члены при $\varepsilon^{\varsigma_{k}}$ и учитывая граничное условие для $u_{\varsigma_{k}}$, получаем краевую задачу для $u_{\varsigma_{k}}$ :

$$
\begin{gathered}
-\Delta u_{\varsigma_{k}}=\sum_{\substack{p, q \\
\varsigma_{p}+\varsigma_{q}=\varsigma_{k}-2}} \lambda_{\varsigma_{p}} u_{\varsigma_{q}} \text { в } \Omega, \\
u_{\varsigma_{k}}=C_{\varsigma_{k}} \text { на } \Gamma_{4}, \\
\frac{\partial u_{\varsigma_{k}}}{\partial \nu}=0 \text { на } \Gamma_{1} \cup \Gamma_{2} \cup \Gamma_{3} .
\end{gathered}
$$

Подставляем (3.58), (3.56) в (1.1) и, учитывая (3.7)-(3.9), собираем члены, имеющие соответствующие степени $\varepsilon$ (в уравнении $-\varepsilon^{\varsigma_{k}-2}$, в граничных условиях $\left.\varepsilon^{\varsigma_{k}-1}\right)$. С учетом периодичности и требованием условия

$$
\frac{\partial v_{\varsigma_{k}}}{\partial x_{1}}\left(\frac{x}{\varepsilon} ; x_{1}\right)=0 \text { при } x_{1}= \pm \frac{\pi}{2}
$$

получаем задачу

$$
\begin{aligned}
& -\Delta_{\xi} v_{\varsigma_{k}}=2 \frac{\partial^{2} v_{\varsigma_{k}-1}}{\partial x_{1} \partial \xi_{1}}+\frac{\partial^{2} v_{\varsigma_{k}-2}}{\partial x_{1}^{2}}
\end{aligned}
$$

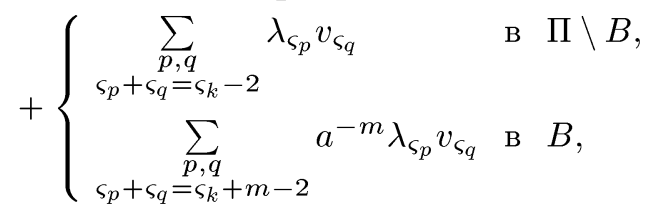

$$
\begin{aligned}
& v_{\varsigma_{k}}=0 \text { на } \gamma, \quad \frac{\partial v_{\varsigma_{k}}}{\partial \xi_{2}}=0 \text { на } \Gamma \text {, } \\
& \frac{\partial v_{\varsigma_{k}}}{\partial \xi_{1}}\left(\xi ; \pm \frac{\pi}{2}\right)=0 \text { при } \xi_{1}= \pm \frac{\pi}{2} \text {. }
\end{aligned}
$$

Если $\varsigma_{i}-1$ или $\varsigma_{i}-2$ не равны $\varsigma_{j}$ для некоторого $j$, то члены с такими индексами в (3.62) равны нулю.

Обозначим через $\mathcal{H}_{\mathrm{Neu}}$ подмножество функций из $C^{\infty}\left[-\frac{\pi}{2}, \frac{\pi}{2}\right]$ таких, что значение их нечетных производных на концах интервала равны нулю. Обозначим через $\mathcal{R}$ подмножество функций из $C^{\infty}(\bar{\Omega})$ таких, что в их разложениях

$$
u(x)=\sum_{k=0}^{\infty} A_{k}\left(x_{1}\right) x_{2}^{k} \text { при } x_{2} \rightarrow 0
$$

коэффициенты $A_{k}$ принадлежат $\mathcal{H}_{\mathrm{Neu}}$. Легко проверить, что функция $u_{0}$ в силу краевой задачи для нее принадлежит $\mathcal{R}$. 
Лемма 3.1. Пусть $C \in \mathcal{H}_{\mathrm{Neu}}, \quad F \in \mathcal{R} ;$ тогда при некотором $\Lambda$ краевая задача

$$
\begin{gathered}
-\Delta u=\lambda_{0} u+F+\Lambda u_{0} \quad \text { в } \quad \Omega, \\
u=C \quad \text { на } \Gamma_{4}, \\
\frac{\partial u}{\partial \nu}=0 \quad \text { на } \quad \Gamma_{1} \cup \Gamma_{2} \cup \Gamma_{3}
\end{gathered}
$$

разрешима в классе $\mathcal{R}$, причем $u \perp u_{0}$ в $L_{2}(\Omega)$.

ДоКАЗАТЕЛЬСТво. Константа $\Lambda$ находится из условия разрешимости, ортогональность $u_{0}$ легко показать, принадлежность $u$ классу $\mathcal{R}$ вытекает в силу краевой задачи для $u$ и принадлежности $F$ классу $\mathcal{R}$.

Из леммы 3.1 непосредственно вытекает утверждение.

Лемма 3.2. Пусть последовательность функций $C_{\varsigma_{k}} \in \mathcal{H}_{\mathrm{Neu}}$. Тогда система рекуррентных задач (3.60) разрешима в классе $\mathcal{R}$ для некоторой последовательности чисел $\lambda_{\varsigma_{k}}$, причем $u_{\varsigma_{k}}$ ортогональны $u_{0}$.

На суммах $U(x, \varepsilon)$ вида $(3.57)$ определим операторы $\mathcal{K}_{\mu}$ следуюшим образом. Коэффициенты ряда $U(x, \varepsilon)$ разложим в ряды при $x_{2} \rightarrow 0$ и перейдем к переменным $\xi_{2}=\frac{x_{2}}{\varepsilon}$. В полученных рядах оставим только члены вида $\varepsilon^{\vartheta} \Phi\left(\xi_{2} ; x_{1}\right)$ при $\vartheta \leqslant \mu$. Этот ряд обозначим $\mathcal{K}_{\mu}(U)$. Здесь $\vartheta, \mu$ не обязательно целые.

Обозначим через $u_{\varepsilon}^{\text {ext }}(x)$ ряд (3.57). Из определения $\mathcal{K}_{\mu}$ вытекает справедливость следующего утверждения.

Лемма 3.3. Пусть $C_{\varsigma_{k}} \in \mathcal{H}_{\mathrm{Neu}}, u_{\varsigma_{k}} \in \mathcal{R}, \lambda_{\varsigma_{k}}$-решения задач (3.60). Тогда для любого $N$

$$
\mathcal{K}_{N}\left(u_{\varepsilon}^{\text {ext }}(x)\right)=\sum_{k=1}^{N} \varepsilon^{\varsigma_{k}} V_{\varsigma_{k}}\left(\xi_{2} ; x_{1}\right) ;
$$

здесь:

1) $V_{\varsigma_{k}}\left(\xi_{2} ; x_{1}\right)$ - многочлены порядка $\left[\varsigma_{k}\right]$ по $\xi_{2}$, коэффициенты которых являются функииями из $\mathcal{H}_{\mathrm{Neu}}$;

2) для $V_{\varsigma_{k}}\left(\xi_{2} ; x_{1}\right)$ выполнено равенство

$$
V_{\varsigma_{k}}\left(\xi_{2} ; x_{1}\right)=\widetilde{V}_{\varsigma_{k}}\left(\xi_{2} ; x_{1}\right)+C_{\varsigma_{k}}\left(x_{1}\right)
$$

где $\widetilde{V}_{\varsigma_{k}}\left(\xi_{2} ; x_{1}\right)$ не зависит от $C_{\varsigma_{j}}$ для всех $j \geqslant k$ и $\widetilde{V}_{\varsigma_{k}}$ являются формальными асимптотическими решениями уравнений (3.62) при $\xi_{2} \rightarrow \infty$, а в правой части уравнения функиии $v_{\varsigma_{s}}$ заменены на многочлены $V_{\varsigma_{s}}$.

Очевидно, что если $V_{\varsigma_{k}}\left(\xi_{2} ; x_{1}\right)$ - многочлен по $\xi_{2}$, коэффициенты которого являются функциями из $\mathcal{H}_{\mathrm{Neu}}$, то

$$
\frac{\partial V_{\varsigma_{k}}}{\partial x_{1}}\left(\frac{x_{2}}{\varepsilon} ; x_{1}\right)=0 \quad \text { при } \quad x_{1}= \pm \frac{\pi}{2} .
$$


Рассмотрим задачи

$$
\begin{gathered}
\Delta Y=F \text { в } \Pi, \\
Y=0 \text { при } \xi_{1}= \pm \frac{\pi}{2}, \\
Y=0 \text { на } \gamma, \quad \frac{\partial Y}{\partial \xi_{2}}=0 \text { на } \Gamma, \\
\Delta Y=F \text { в } \Pi, \\
\frac{\partial Y}{\partial \xi_{1}}=0 \text { при } \xi_{1}= \pm \frac{\pi}{2}, \\
Y=0 \text { на } \gamma, \quad \frac{\partial Y}{\partial \xi_{2}}=0 \text { на } \Gamma .
\end{gathered}
$$

Лемма 3.4. Пусть $e^{\varpi \xi_{2}} F \in L_{2}(\Pi) ;$ тогда существуют единственные решения задач (3.65) и (3.66) с конечным интегралом Дирихле, $ъ>0$.

ДокАЗАТЕЛЬСТво сводится, фактически, к обычной схеме (см., например, [60]). Небольшие изменения возникают в связи с неограниченностью области, для этого используется вариант неравенства Харди (см. аналог неравенства в [71, с. 11]):

$$
\int_{\Pi} \frac{1}{\left(1+\xi_{2}\right)^{2}}|Y|^{2} d \xi \leqslant K \int_{\Pi}\left|\nabla_{\xi} Y\right|^{2} d \xi \quad \forall Y: Y=0 \text { на } \gamma .
$$

ЛЕмма 3.5. Пусть $0<\varpi<2, e^{\varpi \xi_{2}} F \in L_{2}(\Pi) ;$ тогда решение задачи (3.65) с конечным интегралом Дирихле таково, что $e^{\varpi \xi_{2}} Y \in H^{1}(\Pi)$.

ЛЕмма 3.6. Пусть $0<\varpi<2, e^{\varpi \xi_{2}} F \in L_{2}(\Pi) ;$ тогда решение задачи (3.66) с конечным интегралом Дирихле представимо в виде

$$
Y(\xi)=C+y(\xi)
$$

әде $C$ - некоторая постоянная, а $e^{\varpi \xi_{2}} y \in H^{1}(\Pi)$.

ДОКАЗАТЕЛЬСТВА последних двух лемм являются конкретизацией доказательств общих теорем об эллиптических краевых задачах в цилиндрических областях (см. [72, гл. 2, 5]). Необходимо лишь сделать предварительное построение. Рассмотрим в полуполосе $\widetilde{\Pi}=\left\{\xi \in \Pi: \xi_{2}>a\right\}$ следующие задачи:

$$
\begin{gathered}
\Delta \tilde{Y}=F \text { в } \widetilde{\Pi}, \\
\tilde{Y}=0 \text { при } \xi_{1}= \pm \frac{\pi}{2}, \\
\tilde{Y}=K \text { на } \widetilde{\gamma}, \\
\Delta \widetilde{Y}=F \quad \text { в } \widetilde{\Pi}, \\
\frac{\partial \widetilde{Y}}{\partial \xi_{1}}=0 \text { при } \xi_{1}= \pm \frac{\pi}{2}, \\
\tilde{Y}=K \text { на } \widetilde{\gamma},
\end{gathered}
$$

где $\widetilde{\gamma}=\left\{\xi \in \Pi: \xi_{2}=a\right\}$. Утверждения лемм имеют место для решений $\tilde{Y}$ задач (3.67) и (3.68), а решения задач (3.65) и (3.66) получаются как сумма соответствующих решений $\widetilde{Y}$ и решений задач в прямоугольнике $\Pi \backslash \widetilde{\Pi}$ и, следовательно, обладают теми же асимптотическими свойствами при $\xi_{2} \rightarrow+\infty$. 
Теперь введем следующие классы функций:

$$
\begin{aligned}
A_{s}^{\text {even }}= & \left\{e^{\varpi \xi_{2}} f \in H^{s}(\Pi), f \text { - четная и } \pi \text {-периодическая по } \xi_{1},\right. \\
& \left.\frac{\partial f}{\partial \xi_{1}}\left( \pm \frac{\pi}{2}, \xi_{2}\right)=0\right\}, \\
A_{s}^{\text {odd }}= & \left\{e^{\varpi \xi_{2}} f \in H^{s}(\Pi), f \text { - нечетная и } \pi \text {-периодическая по } \xi_{1},\right. \\
& \left.f\left( \pm \frac{\pi}{2}, \xi_{2}\right)=0\right\} .
\end{aligned}
$$

Лемма 3.7. Пусть $F \in A_{0}^{\text {odd } ; ~ т о г д а ~ с у щ е с т в у е т ~ р е ш е н и е ~ к р а е в о и ̆ ~ з а д а-~}$ чи (3.65) из класса $A_{1}^{\text {odd }}$.

ДОКАЗАТЕЛЬСТво вытекает непосредственно из леммы 3.5 с учетом четности функции из условий задачи.

Лемма 3.8. Пусть $F \in A_{0}^{\text {even; }}$ тогда существует решение краевой задачи (3.66), представимое в виде

$$
Y=C+y
$$

əде $y \in A_{1}^{\text {even }}, C$ - некоторая константа.

ДОКАЗАТЕЛЬСТВО вытекает непосредственно из леммы 3.6 с учетом нечетности функции из условий задачи.

Лемма 3.9. Пусть $F$ представимо в виде $F=P_{t}+f, P_{t}-$ многочлен порядка $t$ и $P_{t+2}$ - многочлен порядка $t+2$ такой, что $-P_{t+2}^{\prime \prime}=P_{t}, f \in A_{0}^{\text {even }}$. Тогда существует решение краевой задачи (3.66), представимое в виде

$$
Y=P_{t+2}+C+y,
$$

где $y \in A_{1}^{\text {even }}, C$ - некоторая константа.

ДокАЗАТЕЛьСТВо вытекает из предыдущей леммы.

Обозначим через $\mathcal{H}_{\text {Dir }}$ подмножество функций из $C^{\infty}\left[-\frac{\pi}{2}, \frac{\pi}{2}\right]$ таких, что значение их четных производных на конщах отрезка равны нулю. Из определения классов $\mathcal{H}_{\mathrm{Neu}}$ и $\mathcal{H}_{\text {Dir }}$ следует, что если $g \in \mathcal{H}_{\mathrm{Neu}}$, то $g^{\prime} \in \mathcal{H}_{\text {Dir }}$, и наоборот.

Для дальнейшего анализа понадобятся еще такие классы функций:

$$
\begin{gathered}
\mathcal{A}^{k}=\left\{V\left(\xi ; x_{1}\right): V\left(\xi ; x_{1}\right)=\sum_{j=1}^{J}\left(\alpha_{j}\left(x_{1}\right) Y_{j}(\xi)+\widetilde{\alpha}_{j}\left(x_{1}\right) \widetilde{Y}_{j}(\xi)\right),\right. \\
\left.Y_{j} \in A_{k}^{\text {odd }}, \alpha_{j} \in \mathcal{H}_{\mathrm{Neu}}, \widetilde{Y}_{j} \in A_{k}^{\text {even }}, \widetilde{\alpha}_{j} \in \mathcal{H}_{\mathrm{Dir}}\right\},
\end{gathered}
$$

$J$ - произвольное.

Отметим свойства функций из этих классов:

1) если $V \in \mathcal{A}^{1}$, то $\frac{\partial^{2} V}{\partial \xi_{1} \partial x_{1}} \in \mathcal{A}^{0}, \frac{\partial^{2} V}{\partial x_{1}^{2}} \in \mathcal{A}^{1}$;

2) $\frac{\partial}{\partial x_{1}} V\left(\frac{x}{\varepsilon} ; x_{1}\right)=0$ при $x_{1}= \pm \frac{\pi}{2}$. 
Лемма 3.10. Пусть $P_{t}\left(\xi_{2} ; x_{1}\right)$ и $P_{t+2}\left(\xi_{2} ; x_{1}\right)$ - многочлены по переменной $\xi_{2}$ (порядка $t$ и $t+2$ соответственно), коэффициенты которых являются функциями из $\mathcal{H}_{\mathrm{Neu}}$, причем $-\frac{\partial^{2} P_{t+2}}{\partial \xi_{2}^{2}}=P_{t}, \quad F$ представимо в виде $F=P_{t}+f$, где $f \in \mathcal{A}_{0}$. Тогда существует решение краевой задачи

$$
\begin{gathered}
-\Delta_{\xi} Y=F \quad \text { в } \Pi, \\
Y=0 \quad \text { на } \gamma, \quad \frac{\partial Y}{\partial \xi_{2}}=0 \quad \text { на } \quad \Gamma, \\
\frac{\partial Y}{\partial \xi_{1}}\left(\xi ; \pm \frac{\pi}{2}\right)=0 \quad \text { nри } \quad \xi_{1}= \pm \frac{\pi}{2},
\end{gathered}
$$

представимое в виде $Y=P_{t+2}+C+y$, где $y \in \mathcal{A}_{1}$, a $C \in \mathcal{H}_{\mathrm{Neu}}$.

ДокАЗАТЕЛЬСТво следует из лемм 3.9 и 3.7 и определения классов $\mathcal{A}^{k}$.

Обозначим через $u_{\varepsilon}^{\text {ext }}(x)$ ряд (3.57), а через $u_{\varepsilon}^{\text {int }}-$ ряд (3.58).

ТЕОРема 3.2. Существуют функиии $v_{\varsigma_{k}}$, являющиеся решениями задач (3.62) и представимые в виде $v_{\varsigma_{k}}=V_{\varsigma_{k}}+w_{\varsigma_{k}}$, где $V_{\varsigma_{k}}\left(\xi_{2} ; x_{1}\right)$ - многочлен порядка $\left[\varsigma_{k}\right]$ по $\xi_{2}$, коэффичиенты которого являются функииями из $\mathcal{H}_{\mathrm{Neu}}$, $w_{\varsigma_{k}} \in \mathcal{A}^{1}, \quad C_{\varsigma_{k}} \in \mathcal{H}_{\mathrm{Neu}}, \quad u_{\varsigma_{k}} \in C^{\infty}(\bar{\Omega})$ и постоянные $\lambda_{\varsigma_{k}}$, удовлетворяющие задачам (3.60), такие, что для любого $k$

$$
\mathcal{K}_{\varsigma_{k}}\left(u_{\varepsilon}^{\mathrm{ext}}(x)\right)=\sum_{j=1}^{k} \varepsilon^{\varsigma_{j}} v_{\varsigma_{j}}\left(\xi ; x_{1}\right), \quad \xi_{2} \rightarrow 0,
$$

с точностью до әкспоненциально мальх членов.

ДОКАЗАТЕЛЬСТВО проводим по индукции. Положив $C_{\varsigma_{1}}=-\alpha_{0}$ и определив $v_{\varsigma_{1}}, u_{\varsigma_{1}}, \lambda_{\varsigma_{1}}$ аналогично тому, как сделано в п. 3.1 , получаем справедливость утверждения теоремы для $k=1$.

Предположим, что утверждение теоремы верно для $k \leqslant \mathcal{M}-1$.

По лемме 3.3 и предположению индукции имеем

$$
\mathcal{K}_{\varsigma_{\mathcal{M}}}\left(u_{\varepsilon}^{\mathrm{ext}}(x)\right)=\sum_{j=1}^{\mathcal{M}-1} \varepsilon^{\varsigma_{j}} v_{\varsigma_{j}}\left(\xi ; x_{1}\right)+\varepsilon^{\varsigma_{\mathcal{M}}}\left(\widetilde{V}_{\varsigma_{\mathcal{M}}}\left(\xi_{2} ; x_{1}\right)+C_{\varsigma_{\mathcal{M}}}\left(x_{1}\right)\right), \quad \xi_{2} \rightarrow 0
$$

где $C_{\varsigma_{\mathcal{M}}} \in \mathcal{H}_{\mathrm{Neu}}, \tilde{V}_{\varsigma_{\mathcal{M}}}\left(\xi_{2} ; x_{1}\right)$ - многочлен порядка [ $\left.\varsigma_{\mathcal{M}}\right]$ по $\xi_{2}$, коэффициенты которого являются функциями из $\mathcal{H}_{\mathrm{Neu}}$. Причем $\widetilde{V}_{\varsigma_{\mathcal{M}}}$ не зависит от $C_{\varsigma_{j}}$ для всех $j \geqslant \mathcal{M}$ и является формальным асимптотическим решением уравнения (3.62) при $\xi_{2} \rightarrow \infty$, где в правой части функции $v_{\varsigma_{s}}$ заменены на соответствуюшие многочлены $V_{\varsigma_{s}}$.

Из леммы 3.10 следует, что сушествует решение $v_{\varsigma \mathcal{M}}$ задачи $(3.62)$, представимоев виде $v_{\varsigma_{\mathcal{M}}}=\widetilde{V}_{\varsigma_{\mathcal{M}}}+c_{\varsigma_{\mathcal{M}}}+w_{\varsigma_{\mathcal{M}}}$, где $\widetilde{V}_{\varsigma_{\mathcal{M}}}\left(\xi_{2} ; x_{1}\right)$-многочлен порядка $\left[\varsigma_{\mathcal{M}}\right]$ по $\xi_{2}$, коэффициенты которого являются функциями из $\mathcal{H}_{\mathrm{Neu}}, w_{\varsigma_{k}} \in \mathcal{A}^{1}$. Следовательно,

$$
v_{\varsigma_{\mathcal{M}}} \sim\left(\widetilde{V}_{\varsigma_{\mathcal{M}}}\left(\xi_{2} ; x_{1}\right)+c_{\varsigma_{\mathcal{M}}}\left(x_{1}\right)\right), \quad \xi_{2} \rightarrow 0
$$


с точностью до экспоненциально убывающих членов. Положив $C_{\varsigma_{\mathcal{M}}} \equiv c_{\varsigma_{\mathcal{M}}}$, получаем утверждение теоремы для $k=\mathcal{M}$. Теорема доказана.

Из утверждения теоремы 3.2 и из (3.64), в частности, следует справедливость равенства (3.61)

Обозначим частичные суммы рядов $(3.56)-(3.58)$ соответственно

$$
\begin{gathered}
\tilde{\lambda}_{\varepsilon}^{\mathcal{N}}=\lambda_{0}+\sum_{\substack{j \\
0<\varsigma_{j} \leqslant \mathcal{N}}} \varepsilon^{\varsigma_{j}} \lambda_{\varsigma_{j}}, \\
u_{\varepsilon}^{\mathcal{N}}=u_{0}+\sum_{\substack{j \\
0<\varsigma_{j} \leqslant \mathcal{N}}} \varepsilon^{\varsigma_{j}} u_{\varsigma_{j}}, \\
v_{\varepsilon}^{\mathcal{N}}=\sum_{\substack{j \\
0<\varsigma_{j} \leqslant \mathcal{N}}} \varepsilon^{\varsigma_{j}} v_{\varsigma_{j}} .
\end{gathered}
$$

СЛЕДСТвИЕ 3.1. При $\varepsilon^{\beta}<x_{2}<2 \varepsilon^{\beta}\left(\varepsilon^{\beta-1}<\xi_{2}<2 \varepsilon^{\beta-1}\right), 0<\beta<1$, справедливы равенства

$$
u_{\varepsilon}^{\mathcal{N}}(x)=v_{\varepsilon}^{\mathcal{N}}\left(\frac{x}{\varepsilon} ; x_{1}\right)+O\left(\varepsilon^{\beta(\mathcal{N}+1)}\right) .
$$

ДоКАЗАТЕЛЬСТво. Имеем

$$
\begin{aligned}
u_{\varepsilon}^{\mathcal{N}}-v_{\varepsilon}^{\mathcal{N}} & =u_{\varepsilon}^{\mathcal{N}}-\mathcal{K}_{\mathcal{N}}\left(u_{\varepsilon}^{\mathcal{N}}\right)+\mathcal{K}_{\mathcal{N}}\left(u_{\varepsilon}^{\mathcal{N}}\right)-\mathcal{K}_{\mathcal{N}}\left(u_{\varepsilon}^{\text {ext }}\right) \\
& =u_{\varepsilon}^{\mathcal{N}}-\mathcal{K}_{\mathcal{N}}\left(u_{\varepsilon}^{\mathcal{N}}\right)=O\left(\varepsilon^{\mathcal{N}} x_{2}+x_{2}^{\mathcal{N}+1}\right)=O\left(\varepsilon^{\beta(\mathcal{N}+1)}\right),
\end{aligned}
$$

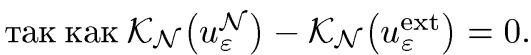

Далее мы покажем малость невязки. Используя теоремы из $\S 2$, обоснуем наши формальные построения, приведенные в п. 3.1.

Рассмотрим срезающую функцию $\chi(t), 0<\chi<1$, которая равна нулю при $t<1$ и равна единице при $t>2$. Определим функцию $\chi_{\beta}, \Upsilon(x)=\chi\left(\frac{x_{2}}{\Upsilon \varepsilon_{\varepsilon}}\right)$, она равна нулю при $x_{2}<\Upsilon \varepsilon^{\beta}$ и равна единище при $x_{2}>2 \Upsilon \varepsilon^{\beta}$, где $0<\beta<1$.

ЛЕмма 3.11. Функиия $\widetilde{u}_{\varepsilon}^{\mathcal{N}}=u_{\varepsilon}^{\mathcal{N}} \chi_{\beta, \Upsilon}+v_{\varepsilon}^{\mathcal{N}}\left(1-\chi_{\beta, \Upsilon)}\right.$ является решением краевой задачи

$$
\begin{gathered}
-\Delta \widetilde{u}_{\varepsilon}^{\mathcal{N}}=\widetilde{\lambda}_{\varepsilon}^{\mathcal{N}} \rho_{\varepsilon} \widetilde{u}_{\varepsilon}^{\mathcal{N}}+f_{\varepsilon}^{\mathcal{N}} \quad n p u \quad x \in \Omega, \\
\widetilde{u}_{\varepsilon}^{\mathcal{N}}=0 \quad n p u \quad x \in \gamma_{\varepsilon}, \\
\frac{\partial \widetilde{u}_{\varepsilon}^{\mathcal{N}}}{\partial \nu}=0 \quad n p u \quad x \in \Gamma_{\varepsilon} \cup \Gamma_{1} \cup \Gamma_{2} \cup \Gamma_{3},
\end{gathered}
$$

əде $\rho_{\varepsilon}$ задана формулой (1.2),

$$
\left\|f_{\varepsilon}^{\mathcal{N}}\right\|_{0}=O\left(\varepsilon^{\beta \mathcal{N}-4}\right)
$$

$$
\widetilde{u}_{\varepsilon}^{\mathcal{N}} \rightarrow u_{0} \quad \text { сильно в } L_{2}(\Omega) \text { при } \quad \varepsilon \rightarrow 0 .
$$


ДоКАЗАТЕЛЬСтво. Заметим, что в силу краевых условий задач (3.62), (3.60) и (3.61) функция $\widetilde{u}_{\varepsilon}^{\mathcal{N}}$ удовлетворяет краевым условиям задачи (1.1). Подействовав на функцию $\widetilde{u}_{\varepsilon}^{\mathcal{N}}$ оператором $\left(-\Delta-\widetilde{\lambda}_{\varepsilon}^{\mathcal{N}} \rho_{\varepsilon}\right)$, получаем

$$
f_{\varepsilon}^{\mathcal{N}}=I_{1}+I_{2}+I_{3}
$$

где

$$
\begin{gathered}
I_{1}=\chi_{\beta, \Upsilon}\left(\Delta u_{\varepsilon}^{\mathcal{N}}-\widetilde{\lambda}_{\varepsilon}^{\mathcal{N}} u_{\varepsilon}^{\mathcal{N}}\right), \\
I_{2}=\left(1-\chi_{\beta, \Upsilon}\right)\left(\Delta v_{\varepsilon}^{\mathcal{N}}-\widetilde{\lambda}_{\varepsilon}^{\mathcal{N}} \rho_{\varepsilon} v_{\varepsilon}^{\mathcal{N}}\right), \\
I_{3}=\varepsilon^{-\beta} \frac{d \chi_{\beta, \Upsilon}}{d x_{2}}\left(\frac{\partial u_{\varepsilon}^{\mathcal{N}}}{\partial x_{2}}-\frac{\partial v_{\varepsilon}^{\mathcal{N}}}{\partial x_{2}}\right)+\varepsilon^{-2 \beta} \frac{d^{2} \chi_{\beta, \Upsilon}}{d x_{2}^{2}}\left(u_{\varepsilon}^{\mathcal{N}}-v_{\varepsilon}^{\mathcal{N}}\right) .
\end{gathered}
$$

В силу уравнений задачи (3.60) имеем $\left\|I_{1}\right\|_{0}=o\left(\varepsilon^{\mathcal{N}}\right)$, в силу уравнений задачи $(3.62)-\left\|I_{2}\right\|_{0}=O\left(\varepsilon^{\beta \mathcal{N}-4}\right)$. Из следствия 3.1 (условия согласования внутреннего и внешнего разложений) и того, что носитель $I_{3}$ лежит в полосе $\varepsilon^{\beta}<x_{2}<2 \varepsilon^{\beta}$ выводим $\left\|I_{3}\right\|_{0}=O\left(\varepsilon^{\beta\left(\mathcal{N}-\frac{1}{2}\right)}\right)$. Учитывая сказанное вьше, получаем $(3.75)$.

Сходимость $(3.76)$ следует из структуры $\widetilde{u}_{\varepsilon}^{\mathcal{N}}$. Лемма доказана.

Этим мы завершили построение формальных асимптотик собственных значений и собственных функций. Теперь перейдем к строгому обоснованию построенных асимптотик.

Учитывая, что $\left\|\widetilde{u}_{\varepsilon}^{\mathcal{N}}\right\|_{0} \rightarrow 1$ при $\varepsilon \rightarrow 0$, и применяя теорему $\left.2.3,1\right)$ для $\lambda=\widetilde{\lambda}_{\varepsilon}^{\mathcal{N}}$, $u=\widetilde{u}_{\varepsilon}^{\mathcal{N}}$ и $f=f_{\varepsilon}^{\mathcal{N}}$. Заключаем, что

$$
\left|\widetilde{\lambda}_{\varepsilon}^{\mathcal{N}}-\lambda_{\varepsilon}\right|=O\left(\varepsilon^{\beta \mathcal{N}-4}\right)
$$

В силу произвольности в выборе $\mathcal{N}$ асимптотика $\lambda_{\varepsilon}$ обоснована.

Перейдем к обоснованию построенной асимптотики собственной функции. Напомним, что по теореме о сходимости $u_{\varepsilon} \rightarrow u_{0}$ сильно в $L_{2}(\Omega)$ при $\varepsilon \rightarrow 0$. Следовательно, в силу (3.76)

$$
\left\|\widetilde{u}_{\varepsilon}^{\mathcal{N}}-u_{\varepsilon}\right\|_{0} \rightarrow 0 \text { при } \varepsilon \rightarrow 0,
$$

где $u_{\varepsilon}-$ решение задачи (1.1) Из представления

$$
\widetilde{u}_{\varepsilon}^{\mathcal{N}}=u_{\varepsilon} \int_{\Omega} u_{\varepsilon} \widetilde{u}_{\varepsilon}^{\mathcal{N}} d x+u_{\varepsilon}^{\perp}, \quad u_{\varepsilon}^{\perp} \perp u_{\varepsilon},
$$

и сходимости (3.78) следует, что

$$
\widetilde{u}_{\varepsilon}^{\mathcal{N}}=T_{\varepsilon}^{\mathcal{N}} u_{\varepsilon}+u_{\varepsilon}^{\perp}, \quad T_{\varepsilon}^{\mathcal{N}}=1+o(1) .
$$

Используя (3.77), (3.79) и лемму 3.11 , заключаем, что функция $u_{\varepsilon}^{\perp}$ является решением краевой задачи

$$
\begin{gathered}
-\Delta u_{\varepsilon}^{\perp}=\tilde{\lambda}_{\varepsilon}^{\mathcal{N}} \rho_{\varepsilon} u_{\varepsilon}^{\perp}+F_{\varepsilon}^{\mathcal{N}} \text { при } x \in \Omega, \\
u_{\varepsilon}^{\perp}=0 \text { при } x \in \gamma_{\varepsilon}, \\
\frac{\partial u_{\varepsilon}^{\perp}}{\partial \nu}=0 \text { при } x \in \Gamma_{\varepsilon} \cup \Gamma_{1} \cup \Gamma_{2} \cup \Gamma_{3},
\end{gathered}
$$


где

$$
F_{\varepsilon}^{\mathcal{N}}=f_{\varepsilon}^{\mathcal{N}}+\rho_{\varepsilon} T_{\varepsilon} u_{\varepsilon}\left(\widetilde{\lambda}_{\varepsilon}^{\mathcal{N}}-\lambda_{\varepsilon}\right)=O\left(\varepsilon^{\beta \mathcal{N}-4-m}\right)
$$

Из соотношений $(3.80),(3.81)$ и утверждения 2$)$ теоремы 2.3 следует, что

$$
\left\|u_{\varepsilon}^{\perp}\right\|_{0}=O\left(\varepsilon^{\beta \mathcal{N}-4-m}\right) .
$$

И наконец, из $(3.79),(3.82)$ и произвольности в выборе $\mathcal{N}$ и $\Upsilon$ вытекает обоснование построенной асимптотики.

Теорема 3.1 доказана полностью.

\section{§4. Кратные собственные значения}

В этом параграфе мы рассмотрим случай, когда собственные значения предельной задачи имеют кратность два. В этом случае также удается построить асимптотики собственных значений и собственных функций исходной задачи. Для собственных значений, имеющих бо́льшую кратность, можно повторить процедуру построения почти дословно.

4.1. Формальный асимптотический анализ. Пусть предельная задача (1.3) имеет собственное значение $\lambda_{0}$ кратности два.

Обозначим две собственные функции этой задачи, ортонормированные в $L_{2}(\Omega)$ и соответствующие собственному значению $\lambda_{0}$, через $u_{0}^{(1)}$ и $u_{0}^{(2)}$. Будем также предполагать, что выполнено дополнительное условие ортогональности

$$
\int_{\Gamma_{4}} \frac{\partial u_{0}^{(1)}}{\partial \nu} \frac{\partial u_{0}^{(2)}}{\partial \nu} d s=0
$$

Дополнительно для простоты изложения будем считать, что

$$
\int_{-\frac{\pi}{2}}^{\frac{\pi}{2}}\left(\frac{\partial u_{0}^{(1)}}{\partial x_{2}}\right)^{2} d x_{1} \neq \int_{-\frac{\pi}{2}}^{\frac{\pi}{2}}\left(\frac{\partial u_{0}^{(2)}}{\partial x_{2}}\right)^{2} d x_{1}
$$

Напомним, что в соответствии с теоремой 2.2 существуют два собственных значения исходной задачи с учетом совокупной кратности, которые сходятся к $\lambda_{0}$, и две собственные функции, сходящиеся к линейным комбинациям собственных функций предельной задачи. Как будет показано ниже, условие (4.2) гарантирует, что эти два собственных значения различны (т. е. являются простыми), условие (4.1) гарантирует, что соответствующие собственные функции будут сходиться к собственным функциям $u_{0}^{(\theta)}, \theta=1,2$, а не к их линейным комбинациям. Таким образом, условие (4.1) определяет правильный выбор главных членов асимптотик для $u_{\varepsilon}^{(\theta)}$.

Везде далее предполагается, что $\theta$ принимает значение 1 или 2. Аналогично случаю простого собственного значения будем искать внешнее разложение в виде

$$
u_{\varepsilon}^{(\theta)}(x)=u_{0}^{(\theta)}(x)+\ldots
$$


внутреннее разложение - в виде

$$
u_{\varepsilon}^{(\theta)}(x)=\varepsilon v_{1}^{(\theta)}\left(\frac{x}{\varepsilon} ; x_{1}\right)+\ldots,
$$

а разложение собственных значений - в виде

$$
\lambda_{\varepsilon}^{(\theta)}=\lambda_{0}+\varepsilon \lambda_{1}^{(\theta)}+\ldots
$$

Обозначим

$$
\alpha_{0}^{(\theta)}\left(x_{1}\right)=\left.\frac{\partial u_{0}^{(\theta)}}{\partial x_{2}}\right|_{x_{2}=0} .
$$

Отметим, что, как и в случае простого собственного значения, имеет место равенство

$$
\left(\alpha_{0}^{(\theta)}\right)^{\prime}\left( \pm \frac{\pi}{2}\right)=0
$$

Подставляя разложения в исходную задачу и учитывая условие согласования, находим задачу для $v_{1}^{(\theta)}$ :

$$
\begin{gathered}
\Delta_{\xi} v_{1}^{(\theta)}=0 \text { в } \Pi, \\
v_{1}^{(\theta)}=0 \text { на } \gamma, \quad \frac{\partial v_{1}^{(\theta)}}{\partial \xi_{2}}=0 \text { на } \Gamma, \\
\frac{\partial v_{1}^{(\theta)}}{\partial \xi_{1}}\left(\xi ; \pm \frac{\pi}{2}\right)=0 \text { при } \xi_{1}= \pm \frac{\pi}{2}, \\
v_{1}^{(\theta)} \sim \alpha_{0}^{(\theta)} \xi_{2} \text { при } \xi_{2} \rightarrow+\infty .
\end{gathered}
$$

Задача (4.5) имеет тот же вид, что и задача (3.10). Как уже отмечалось, решение такой задачи существует, его можно выписать явно:

$$
v_{1}^{(\theta)}\left(\xi ; x_{1}\right)=\alpha_{0}^{(\theta)}\left(x_{1}\right) X(\xi)
$$

где функция $X(\xi)$ задана формулой $(3.12)$, поэтому функция $v_{1}^{(\theta)}\left(\xi ; x_{1}\right)$ является $\pi$-периодической по $\xi_{1}$ и четной по $\xi_{1}$, а ее асимптотика при $\xi_{2} \rightarrow+\infty$ выглядит следуюшим образом:

$$
v_{1}^{(\theta)}(\xi)=\alpha_{0}^{(\theta)}\left(x_{1}\right)\left[\left(\xi_{2}-\ln \sin a\right)+O\left(e^{-2 \xi_{2}}\right)\right] .
$$

Отметим, что благодаря (4.4) выполняется равенство

$$
\frac{\partial v_{1}^{(\theta)}}{\partial x_{1}}\left(\xi ; x_{1}\right)=0 \quad \text { при } \quad x_{1}= \pm \frac{\pi}{2},
$$

и, следовательно, в силу $(3.8),(3.9),(4.5)$ и (4.8) имеем

$$
\frac{\partial v_{1}^{(\theta)}}{\partial \nu}\left(\frac{x}{\varepsilon} ; x_{1}\right)=0 \text { на } \Gamma_{2} \cup \Gamma_{3} .
$$


Как и ранее, устраняя невязку в граничных условиях для функции $u_{0}^{(\theta)}(x)$, мы построили $v_{1}^{(\theta)}\left(\xi ; x_{1}\right)$, при этом появилась новая невязка меньшего порядка. Эта невязка устраняется введением следуюшего члена во внешнем разложении. Процедура не отличается от той, которую мы использовали в $\S 3$. Пересчитав асимптотику $\varepsilon v_{1}^{(\theta)}$ в бесконечности во внешних переменных с учетом (4.7), заключаем, что внешнее разложение имеет вид

$$
u_{\varepsilon}^{(\theta)}(x)=u_{0}^{(\theta)}(x)+\varepsilon u_{1}^{(\theta)}(x)+\ldots,
$$

где функция $u_{1}^{(\theta)}(x)$ удовлетворяет следуюшей краевой задаче:

$$
\begin{gathered}
-\Delta u_{1}^{(\theta)}=\lambda_{0} u_{1}^{(\theta)}+\lambda_{1}^{(\theta)} u_{0}^{(\theta)} \text { в } \Omega, \\
u_{1}^{(\theta)}=-\alpha_{0}^{(\theta)} \ln \sin a \text { на } \Gamma_{4}, \\
\frac{\partial u_{1}^{(\theta)}}{\partial \nu}(x)=0 \text { на } \Gamma_{1} \cup \Gamma_{2} \cup \Gamma_{3} .
\end{gathered}
$$

Отметим, что, в отличие от случая простого собственного значения, теперь условий разрешимости задачи (4.10) будет два. Умножая уравнение задачи (4.10) на $u_{0}^{(\theta)}$ и применяя формулу Грина, находим первое условие

$$
\lambda_{1}^{(\theta)}=\ln \sin a \int_{-\frac{\pi}{2}}^{\frac{\pi}{2}}\left(\alpha_{0}^{(\theta)}\left(x_{1}\right)\right)^{2} d x_{1},
$$

или

$$
\lambda_{1}^{(\theta)}=\ln \sin a \int_{\Gamma_{4}}\left(\frac{\partial u_{0}^{(\theta)}}{\partial \nu}\right)^{2} d s .
$$

Проверим второе условие разрешимости. Домножим уравнение из (4.10) при $\theta=1$ на функцию $u_{0}^{(2)}(x)$ и проинтегрируем по $\Omega$, применяя формулу Грина:

$$
\begin{aligned}
& \lambda_{0} \int_{\Omega} u_{1}^{(1)}(x) u_{0}^{(2)}(x) d x+\lambda_{1}^{(\theta)} \int_{\Omega} u_{0}^{(1)}(x) u_{0}^{(2)}(x) d x \\
& \quad=-\int_{\Omega} u_{1}^{(1)}(x) \Delta u_{0}^{(2)}(x) d x-\int_{\partial \Omega} \frac{\partial u_{1}^{(1)}}{\partial \nu} u_{0}^{(2)} d s+\int_{\partial \Omega} \frac{\partial u_{0}^{(2)}}{\partial \nu} u_{1}^{(1)} d s \\
& \quad=\lambda_{0} \int_{\Omega} u_{1}^{(1)}(x) u_{0}^{(2)}(x) d x+\ln \sin a \int_{\Gamma_{4}} \frac{\partial u_{0}^{(2)}}{\partial \nu} \frac{\partial u_{0}^{(1)}}{\partial \nu} d s
\end{aligned}
$$

Здесь мы воспользовались тем, что на $\Gamma_{4}$

$$
u_{1}^{(1)}=\frac{\partial u_{0}^{(1)}}{\partial \nu} \text {. }
$$

Ясно, что (4.12) является тождеством, так как из ортогональности функций $u_{0}^{(1)}$ и $u_{0}^{(2)}$ следует, что левая часть этого равенства равна нулю, а из условия (4.1) 
следует, что и правая часть равенства равна нулю. Таким образом, второе условие разрешимости выполнено при $\theta=1$. Аналогично поступим в случае $\theta=2$.

Отметим, что дополнительное условие ортогональности на границе типа (4.1) ранее было использовано в работе [73].

Произвольность выбора функщий $u_{1}^{(\theta)}$ будем фиксировать следующими условиями. Выбираем функции в виде

$$
\begin{aligned}
& u_{1}^{(1)}=\widetilde{u}_{1}^{(1)}+\kappa_{1}^{(1)} u_{0}^{(2)}, \\
& u_{1}^{(2)}=\widetilde{u}_{1}^{(2)}+\kappa_{1}^{(2)} u_{0}^{(1)},
\end{aligned}
$$

где $\widetilde{u}_{1}^{(\theta)}$ удовлетворяет условию ортогональности:

$$
\int_{\Omega} \widetilde{u}_{1}^{(\theta)}(x) u_{0}^{(\eta)}(x) d x=0, \quad \theta, \eta=1,2
$$

Здесь константы $\kappa_{1}^{(\theta)}$ пока произвольны, они будут выбраны однозначно из условия разрешимости краевых задач для следующих членов (а именно, при исследовании задачи для $\left.u_{2}^{(\theta)}\right)$.

Продолжаем процесс построения асимптотик. Так же, как и для простого собственного значения $\lambda_{0}$, вначале рассмотрим случай $0<m<1$. Из определения функции $u_{1}^{(\theta)}(x)$ имеем

$$
\frac{\partial u_{1}^{(\theta)}}{\partial x_{2}}\left(x_{1}, 0\right)=\alpha_{1}^{(\theta)}\left(x_{1}\right)+\kappa_{1}^{(\theta)} \alpha_{0}^{(\eta)}\left(x_{1}\right)
$$

где

$$
\alpha_{1}^{(\theta)}=\frac{\partial \widetilde{u}_{1}^{(\theta)}}{\partial x_{2}}
$$

Здесь и далее предполагается, что $\theta$ принимает значения 1 и 2 , а $\eta=3-\theta$, т.е. $\eta=1$, если $\theta=2$, и, наоборот, $\eta=2$, если $\theta=1$.

Отметим, что благодаря гладкости функции $u_{1}^{(\theta)}$, граничных условий для $u_{1}^{(\theta)}$ и (4.4) мы получаем

$$
\left(\alpha_{1}^{(\theta)}\right)^{\prime}\left( \pm \frac{\pi}{2}\right)=0
$$

Переписывая асимптотику $u_{1}^{(\theta)}(x)$ в нуле в переменных $\left(x_{1}, \xi_{2}\right)$, где $\xi_{2}=\frac{x_{2}}{\varepsilon}$, получаем, что внутреннее разложение должно иметь вид

$$
u_{\varepsilon}^{(\theta)}(x)=\varepsilon v_{1}^{(\theta)}\left(\xi ; x_{1}\right)+\varepsilon^{2} v_{2}^{(\theta)}\left(\xi ; x_{1}\right)+\ldots
$$


где $v_{2}^{(\theta)}\left(\xi ; x_{1}\right)$ является решением задачи

$$
\begin{gathered}
-\Delta_{\xi} v_{2}^{(\theta)}=2 \frac{\partial^{2} v_{1}^{(\theta)}}{\partial x_{1} \partial \xi_{1}} \text { в } \Pi, \\
v_{2}^{(\theta)}=0 \text { на } \gamma, \quad \frac{\partial v_{2}^{(\theta)}}{\partial \xi_{2}}=0 \text { на } \Gamma, \\
\frac{\partial v_{2}^{(\theta)}}{\partial \xi_{1}}\left(\xi ; \pm \frac{\pi}{2}\right)=0 \text { при } \xi_{1}= \pm \frac{\pi}{2}, \\
v_{2}^{(\theta)}(\xi) \sim\left(\alpha_{1}^{(\theta)}\left(x_{1}\right)+\kappa_{1}^{(\theta)} \alpha_{0}^{(\eta)}\left(x_{1}\right)\right) \xi_{2} \quad \text { при } \xi_{2} \rightarrow+\infty .
\end{gathered}
$$

Отличие этой задачи от задачи для $v_{2}$ в случае простого собственного значения состоит в том, что появилась пока неизвестная константа $\kappa_{1}^{(\theta)}$ в асимптотике функции на бесконечности. Как и в случае простого собственного значения, можно проверить непосредственно, что в силу (3.12), (3.44), (3.45), (4.4) и (4.13) функция

$$
v_{2}^{(\theta)}(\xi)=\left(\alpha_{1}^{(\theta)}\left(x_{1}\right)+\kappa_{1}^{(\theta)} \alpha_{0}^{(\eta)}\left(x_{1}\right)\right) X(\xi)-2\left(\alpha_{0}^{(\theta)}\right)^{\prime}\left(x_{1}\right) \tilde{X}(\xi)
$$

является $\pi$-периодическим решением задачи (4.15) с асимптотикой на бесконечности

$$
\begin{aligned}
v_{2}^{(\theta)}(\xi) \sim & \left(\alpha_{1}^{(\theta)}\left(x_{1}\right)+\kappa_{1}^{(\theta)} \alpha_{0}^{(\eta)}\left(x_{1}\right)\right) \xi_{2} \\
& -\left(\alpha_{1}^{(\theta)}\left(x_{1}\right)+\kappa_{1}^{(\theta)} \alpha_{0}^{(\eta)}\left(x_{1}\right)\right) \ln \sin a+\ldots
\end{aligned}
$$

где точками обозначены экспоненциально убывающие члены. Здесь $X(\xi)$ - функция, заданная в (3.12), а $\widetilde{X}(\xi)$ - решение задачи (3.44) с асимптотикой $(3.45)$.

Отметим, что в силу соотношений (4.4), (4.13), четности функции $X(\xi)$ по $\xi_{1}$, нечетности $\widetilde{X}(\xi)$ по $\xi_{1}$, а также выполнения краевого условия $\widetilde{X}\left( \pm \frac{\pi}{2}, \xi_{2}\right)=0$ имеем

$$
\frac{\partial v_{2}^{(\theta)}}{\partial x_{1}}\left( \pm \frac{\pi}{2}, \xi_{2} ; x_{1}\right)=0 \text { при } x_{1}= \pm \frac{\pi}{2}
$$

и, следовательно,

$$
\frac{\partial v_{2}^{(\theta)}}{\partial \nu}\left(\frac{x}{\varepsilon} ; x_{1}\right)=0 \text { на } \Gamma_{2} \cup \Gamma_{3} .
$$

Как и в случае простого собственного значения, для устранения невязки

$$
-\varepsilon^{2}\left(\alpha_{1}^{(\theta)}\left(x_{1}\right)+\kappa_{1}^{(\theta)} \alpha_{0}^{(\eta)}\left(x_{1}\right)\right) \ln \sin a
$$

внешние разложения для собственных функций и асимптотики собственных значений приходится вновь искать в виде

$$
\begin{gathered}
u_{\varepsilon}^{(\theta)}(x)=u_{0}^{(\theta)}(x)+\varepsilon u_{1}^{(\theta)}(x)+\varepsilon^{2} u_{2}^{(\theta)}(x)+\ldots, \\
\lambda_{\varepsilon}^{(\theta)}=\lambda_{0}+\varepsilon \lambda_{1}^{(\theta)}+\varepsilon^{2} \lambda_{2}^{(\theta)}+\ldots
\end{gathered}
$$


Задача для $u_{2}^{(\theta)}(x)$ имеет вид

$$
\begin{gathered}
-\Delta u_{2}^{(\theta)}=\lambda_{0} u_{2}^{(\theta)}+\lambda_{1}^{(\theta)} u_{1}^{(\theta)}+\lambda_{2}^{(\theta)} u_{0}^{(\theta)} \text { в } \Omega, \\
u_{2}^{(\theta)}=-\left(\alpha_{1}^{(\theta)}+\kappa_{1}^{(\theta)} \alpha_{0}^{(\eta)}\right) \ln \sin a \text { на } \Gamma_{4}, \\
\frac{\partial u_{2}^{(\theta)}}{\partial \nu}=0 \text { на } \Gamma_{1} \cup \Gamma_{2} \cup \Gamma_{3} .
\end{gathered}
$$

Заметим, что в отличие от случая простого $\lambda_{0}$, во-первых, граничное условие на $\Gamma_{4}$ в $(4.22)$ однозначно не определено (так как содержит неизвестную $\kappa_{1}^{(\theta)}$ ), а во-вторых, для задачи требуется два условия разрешимости (как мы уже отмечали). С учетом (4.1) получаем, что эти условия определяются равенствами

$$
\begin{aligned}
\lambda_{2}^{(\theta)} & =\ln \sin a \int_{-\frac{\pi}{2}}^{\frac{\pi}{2}}\left(\alpha_{1}^{(\theta)}\left(x_{1}\right)+\kappa_{1}^{(\theta)} \alpha_{0}^{(\eta)}\left(x_{1}\right)\right) \alpha_{0}^{(\theta)}\left(x_{1}\right) d x_{1} \\
& =\ln \sin a \int_{-\frac{\pi}{2}}^{\frac{\pi}{2}} \alpha_{1}^{(\theta)}\left(x_{1}\right) \alpha_{0}^{(\theta)}\left(x_{1}\right) d x_{1}
\end{aligned}
$$

а также

$$
\kappa_{1}^{(\theta)} \lambda_{1}^{(\theta)}=\ln \sin a \int_{-\frac{\pi}{2}}^{\frac{\pi}{2}}\left(\alpha_{1}^{(\theta)}\left(x_{1}\right)+\kappa_{1}^{(\theta)} \alpha_{0}^{(\eta)}\left(x_{1}\right)\right) \alpha_{0}^{(\eta)}\left(x_{1}\right) d x_{1}
$$

Из последнего равенства находим

$$
\kappa_{1}^{(\theta)}=\frac{\int_{-\frac{\pi}{2}}^{\frac{\pi}{2}} \alpha_{1}^{(\theta)}\left(x_{1}\right) \alpha_{0}^{(\eta)}\left(x_{1}\right) d x_{1}}{\int_{-\frac{\pi}{2}}^{\frac{\pi}{2}}\left(\left(\alpha_{0}^{(\theta)}\right)^{2}\left(x_{1}\right)-\left(\alpha_{0}^{(\eta)}\right)^{2}\left(x_{1}\right)\right) d x_{1}}
$$

Таким образом мы определили константы $\lambda_{2}^{(\theta)}$ и $\kappa_{1}^{(\theta)}$.

Произвольность выбора функций $u_{2}^{(\theta)}$ вновь фиксируется следующим образом:

$$
\begin{aligned}
& u_{2}^{(1)}=\widetilde{u}_{2}^{(1)}+\kappa_{2}^{(1)} u_{0}^{(2)}, \\
& u_{2}^{(2)}=\widetilde{u}_{2}^{(2)}+\kappa_{2}^{(2)} u_{0}^{(1)},
\end{aligned}
$$

где $\widetilde{u}_{2}^{(\theta)}$ удовлетворяет условию ортогональности

$$
\int_{\Omega} \widetilde{u}_{2}^{(\theta)}(x) u_{0}^{(\eta)}(x) d x=0, \quad \theta, \eta=1,2
$$

константы $\kappa_{2}^{(\theta)}$ пока произвольны; они будут выбраны однозначно из условия разрешимости краевых задач для $u_{3}^{(\theta)}$. 
Рассмотрим случай $1<m<2$. Как и для простого $\lambda_{0}$, асимптотики строятся в виде

$$
\begin{aligned}
& \lambda_{\varepsilon}^{(\theta)}(x)=\lambda_{0}(x)+\varepsilon \lambda_{1}^{(\theta)}(x)+\varepsilon^{3-m} \lambda_{3-m}^{(\theta)}(x)+\ldots, \\
& u_{\varepsilon}^{(\theta)}(x)=u_{0}^{(\theta)}(x)+\varepsilon u_{1}^{(\theta)}(x)+\varepsilon^{3-m} u_{3-m}^{(\theta)}(x)+\ldots, \\
& u_{\varepsilon}^{(\theta)}(x)=\varepsilon v_{1}^{(\theta)}\left(\xi ; x_{1}\right)+\varepsilon^{3-m} v_{3-m}^{(\theta)}\left(\xi ; x_{1}\right)+\ldots,
\end{aligned}
$$

где задача для $v_{3-m}^{(\theta)}$, по-прежнему, имеет вид

$$
\begin{aligned}
& -\Delta_{\xi} v_{3-m}^{(\theta)}= \begin{cases}0 & \text { в } \Pi \backslash B, \\
a^{-m} \lambda_{0} v_{1}^{(\theta)} & \text { в } B,\end{cases} \\
& v_{3-m}^{(\theta)}=0 \text { на } \gamma, \quad \frac{\partial v_{3-m}^{(\theta)}}{\partial \xi_{2}}=0 \text { на } \Gamma, \\
& \frac{\partial v_{3-m}^{(\theta)}}{\partial \xi_{1}}\left(\xi ; \pm \frac{\pi}{2}\right)=0 \text { при } \xi_{1}= \pm \frac{\pi}{2} .
\end{aligned}
$$

Поэтому функция

$$
v_{3-m}^{(\theta)}\left(\xi ; x_{1}\right)=\alpha_{0}^{(\theta)}\left(x_{1}\right) X_{3-m}(\xi)
$$

удовлетворяет задаче (3.81) и имеет асимптотику

$$
v_{3-m}^{(\theta)}\left(\xi ; x_{1}\right)=\alpha_{0}^{(\theta)}\left(x_{1}\right)\left(\left(\xi_{2}+c_{3-m}\right)+O\left(e^{-2 \xi_{2}}\right)\right) \quad \text { при } \quad \xi_{2} \rightarrow+\infty .
$$

Здесь, напомним, функция $X_{3-m}(\xi)$ - решение задачи $(3.30)$ с асимптотикой на бесконечности (3.31), константа $c_{3-m}$ подсчитана в формуле (3.34). Отметим, что благодаря (4.4) и (4.30) выполняется

$$
\frac{\partial v_{3-m}^{(\theta)}}{\partial x_{1}}\left(\xi ; x_{1}\right)=0 \text { при } x_{1}= \pm \frac{\pi}{2},
$$

и, следовательно, в силу $(3.8),(3.9),(4.29)$ и (4.32) имеем

$$
\frac{\partial v_{3-m}^{(\theta)}}{\partial \nu}\left(\frac{x}{\varepsilon} ; x_{1}\right)=0 \text { на } \Gamma_{2} \cup \Gamma_{3} .
$$

Функция $u_{3-m}^{(\theta)}(x)$ должна быть решением следующей задачи:

$$
\begin{gathered}
-\Delta u_{3-m}^{(\theta)}=\lambda_{0} u_{3-m}^{(\theta)}+\lambda_{3-m}^{(\theta)} u_{0}^{(\theta)} \text { в } \Omega, \\
u_{3-m}^{(\theta)}=c_{3-m} \alpha_{0}^{(\theta)} \text { на } \Gamma_{4}, \\
\frac{\partial u_{3-m}^{(\theta)}}{\partial \nu}=0 \text { на } \Gamma_{1} \cup \Gamma_{2} \cup \Gamma_{3} .
\end{gathered}
$$


Записываем опять оба условия разрешимости. Одно из них дает:

$$
\lambda_{3-m}^{(\theta)}=-\int_{-\frac{\pi}{2}}^{\frac{\pi}{2}} c_{3-m}\left(\alpha_{0}^{(\theta)}\right)^{2} d x_{1} .
$$

Другое условие выполняется в силу (4.1)

Заметим, что в противном случае нам бы пришлось вводить во внешнем разложении функции $u_{\varepsilon}^{(\theta)}$ член $u_{2-m}^{(\theta)}=\kappa_{2-m}^{(\theta)} u_{0}^{(\eta)}$ (аналогично тому, как это пришлось делать при определении $\left.\lambda_{2}^{(\theta)}\right)$. Функция $u_{3-m}^{(\theta)}$ опять определяется в виде

$$
\begin{aligned}
& u_{3-m}^{(1)}=\widetilde{u}_{3-m}^{(1)}+\kappa_{3-m}^{(1)} u_{0}^{(2)}, \\
& u_{3-m}^{(2)}=\widetilde{u}_{3-m}^{(2)}+\kappa_{3-m}^{(2)} u_{0}^{(1)},
\end{aligned}
$$

где $\widetilde{u}_{3-m}^{(\theta)}$ удовлетворяет условию ортогональности

$$
\int_{\Omega} \widetilde{u}_{3-m}^{(\theta)}(x) u_{0}^{(\eta)}(x) d x=0, \quad \theta, \eta=1,2
$$

а константы $\kappa_{3-m}^{(\theta)}$ пока произвольны.

Случай $m=1$ (с учетом рассмотренного выше в этом пункте) полностью аналогичен случаю $m=1$ для простого собственного значения $\lambda_{0}$. А именно, собственные функции имеют асимптотики (4.20) и (4.14), а собственные значения - (4.21), где $u_{2}^{(\theta)}$ - суммы решений задач (4.34) и (4.22) (а $\kappa_{1}^{(\theta)}$ определяются из $\left.(4.25)\right), v_{2}^{(\theta)}$ суммы решений задач (4.29) и (4.15), а собственные числа $\lambda_{2}^{(\theta)}$ - суммы постоянных, определяемых формулами (4.23) и (4.35).

4.2. Построение полной асимптотики. Построим полные асимптотические разложения собственных значений и собственных функций задачи (1.1) и строго обоснуем их.

Как и в случае простого собственного значения, перенумеруем множество $\{1+$ $i+(2-m) j\}_{i, j=0}^{\infty}$ для фиксированного $m$ в порядке возрастания: $1=\varsigma_{1}<\varsigma_{2}<\ldots$. Легко видеть, что $\varsigma_{2}=2$ при $0<m \leqslant 1$, а $\varsigma_{2}=3-m$ при $1<m<2$.

Пусть $0<\beta<1-$ произвольное число. В настоящем пункте проведем доказательство следующего утверждения.

Tеорема 4.1. Асимптотики собственных значений и соответствующих собственных функиий имеют вид

$$
\begin{gathered}
\lambda_{\varepsilon}^{(\theta)}=\lambda_{0}+\sum_{i=1}^{\infty} \varepsilon^{\varsigma_{i}} \lambda_{\varsigma_{i}}^{(\theta)}, \\
\mathcal{U}_{\varepsilon}^{(\theta)}(x)=u_{0}^{(\theta)}(x)+\sum_{i=1}^{\infty} \varepsilon^{\varsigma_{i}} u_{\varsigma_{i}}^{(\theta)}(x), \quad x_{2}>\varepsilon^{\beta}, \\
\mathcal{U}_{\varepsilon}^{(\theta)}(x)=\sum_{i=1}^{\infty} \varepsilon^{\varsigma_{i}} v_{\varsigma_{i}}^{(\theta)}\left(\frac{x}{\varepsilon} ; x_{1}\right), \quad 0 \leqslant x_{2}<\varepsilon^{\beta},
\end{gathered}
$$


в норме пространства Соболева $H^{1}(\Omega)$. Здесь $u_{\varsigma_{k}}^{(\theta)} \in C^{\infty}(\bar{\Omega}), \quad v_{\varsigma_{k}}^{(\theta)}\left(\xi ; x_{1}\right)$ $\pi$-периодические по $\xi_{1}$ функиии с асимптотикой

$$
v_{\varsigma_{i}}^{(\theta)}\left(\xi ; x_{1}\right) \sim P_{\left[\varsigma_{i}\right]}\left(\xi_{2}\right) \quad \text { npu } \quad \xi_{2} \rightarrow \infty
$$

для любого фиксированного $x_{1}$, где $\left[\varsigma_{i}\right]-$ целая часть $\varsigma_{i}, P_{k}\left(\xi_{2}\right)$-многочлен степени $k$,

$$
\begin{gathered}
v_{\varsigma_{1}}^{(\theta)}\left(\xi ; x_{1}\right)=\alpha_{0}^{(\theta)}\left(x_{1}\right) X(\xi), \\
\lambda_{\varsigma_{1}}^{(\theta)}=\ln \sin a \int_{-\frac{\pi}{2}}^{\frac{\pi}{2}}\left(\alpha_{0}^{(\theta)}\left(x_{1}\right)\right)^{2} d x_{1}, \\
\lambda_{\varsigma_{2}}^{(\theta)}=-\frac{a^{-m} \lambda_{0}}{\pi} \int_{-\frac{\pi}{2}}^{\frac{\pi}{2}}\left(\alpha_{0}^{(\theta)}\left(x_{1}\right)\right)^{2} d x_{1} \int_{B} X^{2}(\xi) d \xi \quad n p u \quad 1<m<2, \\
\lambda_{\varsigma_{2}}^{(\theta)}=\ln \sin a \int_{-\frac{\pi}{2}}^{\frac{\pi}{2}} \alpha_{1}^{(\theta)}\left(x_{1}\right) \alpha_{0}^{(\theta)}\left(x_{1}\right) d x_{1} \quad 1<\quad n p u \quad 0<m \leqslant 1, \\
-\delta_{m}^{1} \frac{a^{-m} \lambda_{0}}{\pi} \int_{-\frac{\pi}{2}}^{\frac{\pi}{2}}\left(\alpha_{0}^{(\theta)}\left(x_{1}\right)\right)^{2} d x_{1} \int_{B} X^{2}(\xi) d \xi \quad n p \quad 0 \quad
\end{gathered}
$$

əде $X(\xi)=\operatorname{Re} \ln \left(\sin z+\sqrt{\sin ^{2} z-\sin ^{2} a}\right)-\ln \sin a, \quad z=\xi_{1}+i \xi_{2}, \quad \alpha_{0}^{(\theta)}\left(x_{1}\right)=$ $\left.\frac{\partial u_{0}^{(\theta)}}{\partial x_{2}}\right|_{x_{2}=0}, \quad \alpha_{1}^{(\theta)}\left(x_{1}\right)=\left.\frac{\partial u_{1}^{(\theta)}}{\partial x_{2}}\right|_{x_{2}=0}, \quad u_{1}^{(\theta)}-$ решение задачи $(4.10), \delta_{k}^{l}-$ символ Кронекера.

ЗАМЕчАНИЕ 4.1. Здесь $\mathcal{U}_{\varepsilon}^{(\theta)}(x)=T_{\varepsilon}^{(\theta)} u_{\varepsilon}^{(\theta)}(x)$, константы $T_{\varepsilon}^{(\theta)}=1+o(1)$ при $\varepsilon \rightarrow 0$.

Доказательство теоремы 4.1 почти аналогично доказательству теоремы 3.1 для простого $\lambda_{0}$. Некоторые различия возникают только при обосновании асимптотик. Действительно, обозначим через $\left(u_{\varepsilon}^{(\theta)}\right)^{\text {ext }}(x)$ ряд $(4.37)$, а через $\left(u_{\varepsilon}^{(\theta)}\right)^{\text {int }}(x)-$ ряд (4.38).

ТЕОрема 4.2. Существуют функиии $v_{\varsigma_{k}}^{(\theta)}$, являющиеся решениями задач

$$
\begin{aligned}
& -\Delta_{\xi} v_{\varsigma_{k}}^{(\theta)}=2 \frac{\partial^{2} v_{\varsigma_{k}-1}^{(\theta)}}{\partial x_{1} \partial \xi_{1}}+\frac{\partial^{2} v_{\varsigma_{k}-2}^{(\theta)}}{\partial x_{1}^{2}} \\
& +\left\{\begin{array}{cll}
\sum_{\substack{p, q \\
\varsigma_{p}+\varsigma_{q}=\varsigma_{k}-2}} \lambda_{\varsigma_{p}}^{(\theta)} v_{\varsigma_{q}}^{(\theta)} & \theta & \Pi \backslash B, \\
\sum_{\substack{p, q \\
\varsigma_{p}+\varsigma_{q}=\varsigma_{k}+m-2}} a^{-m} \lambda_{\varsigma_{p}}^{(\theta)} v_{\varsigma_{q}}^{(\theta)} & 8 & B,
\end{array}\right. \\
& v_{\varsigma_{k}}^{(\theta)}=0 \quad \text { нa } \gamma, \quad \frac{\partial v_{\varsigma_{k}}^{(\theta)}}{\partial \xi_{2}}=0 \quad \text { нa } \Gamma, \\
& \frac{\partial v_{\varsigma_{k}}^{(\theta)}}{\partial \xi_{1}}\left(\xi ; \pm \frac{\pi}{2}\right)=0 \quad n p u \quad \xi_{1}= \pm \frac{\pi}{2}
\end{aligned}
$$


u представимые в виде $v_{\varsigma_{k}}^{(\theta)}=V_{\varsigma_{k}}^{(\theta)}+w_{\varsigma_{k}}^{(\theta)}$, где $V_{\varsigma_{k}}^{(\theta)}\left(\xi_{2} ; x_{1}\right)$ - многочлен порядка $\left[\varsigma_{k}\right]$ по $\xi_{2}$, коэффичиенты которого являются функииями из $\mathcal{H}_{\mathrm{Neu}}$, $w_{\varsigma_{k}}^{(\theta)} \in \mathcal{A}^{1}, C_{\varsigma_{k}} \in \mathcal{H}_{\mathrm{Neu}}, u_{\varsigma_{k}}^{(\theta)} \in C^{\infty}(\bar{\Omega})$, и постоянние $\lambda_{\varsigma_{k}}$, удовлетворяющие задачам

$$
\begin{aligned}
& -\Delta u_{\varsigma_{k}}^{(\theta)}=\sum_{\substack{p, q \\
\varsigma_{p}+\varsigma_{q}=\varsigma_{k}-2}} \lambda_{\varsigma_{p}}^{(\theta)} u_{\varsigma_{q}}^{(\theta)} \quad \theta \quad \Omega, \\
& u_{\varsigma_{k}}^{(\theta)}=C_{\varsigma_{k}}^{(\theta)} \quad \text { на } \quad \Gamma_{4}, \\
& \frac{\partial u_{\varsigma_{k}}^{(\theta)}}{\partial \nu}=0 \quad \text { нa } \Gamma_{1} \cup \Gamma_{2} \cup \Gamma_{3},
\end{aligned}
$$

такие, что для любого $k$

$$
\mathcal{K}_{\varsigma_{k}}\left(\left(u_{\varepsilon}^{(\theta)}(x)\right)^{\mathrm{ext}}\right)=\sum_{j=1}^{k} \varepsilon^{\varsigma_{j}} v_{\varsigma_{j}}^{(\theta)}\left(\xi ; x_{1}\right) \quad \text { npu } \quad \xi_{2} \rightarrow 0
$$

с точностью до әкспоненциально малых членов.

Если $\varsigma_{i}-1$ или $\varsigma_{i}-2$ не равнь $\varsigma_{j}$ для некоторого $j$, то члены с такими индексами в (4.40) равны нулю.

СЛЕДСТВИЕ 4.1. При $\varepsilon^{\beta}<x_{2}<2 \varepsilon^{\beta}\left(\varepsilon^{\beta-1}<\xi_{2}<2 \varepsilon^{\beta-1}\right), \quad 0<\beta<1$, справедливы равенства

$$
\left\{u_{\varepsilon}^{(\theta)}\right\}^{\mathcal{N}}(x)=\left\{v_{\varepsilon}^{(\theta)}\right\}^{\mathcal{N}}\left(\frac{x}{\varepsilon} ; x_{1}\right)+O\left(\varepsilon^{\beta(\mathcal{N}+1)}\right)
$$

əде $\left\{u_{\varepsilon}^{(\theta)}\right\}^{\mathcal{N}} u\left\{v_{\varepsilon}^{(\theta)}\right\}^{\mathcal{N}}-$ частичные суммы рядов (4.37) и (4.38) соответственно.

Теорема 4.2 и следствие 4.1 доказываются полностью аналогично теореме 3.2 и следствию 3.1 .

Обозначим через $\widetilde{\lambda}_{\varepsilon}^{(\theta)}$ построенные ряды $(4.36)$, а через $\left\{\widetilde{\lambda}_{\varepsilon}^{(\theta)}\right\}^{\mathcal{N}}-$ их частичные суммы. Из теоремы 4.2 и следствия 4.1 вытекает справедливость следующего аналога леммы 3.11 .

Лемма 4.1. Функиия $\left\{\widetilde{u}_{\varepsilon}^{(\theta)}\right\}^{\mathcal{N}}=\left\{u_{\varepsilon}^{(\theta)}\right\}^{\mathcal{N}} \chi_{\beta, \Upsilon}+\left\{v_{\varepsilon}^{(\theta)}\right\}^{\mathcal{N}}\left(1-\chi_{\beta, \Upsilon)}\right.$ яв.ляется решением краевой задачи

$$
\begin{gathered}
-\Delta\left\{\widetilde{u}_{\varepsilon}^{(\theta)}\right\}^{\mathcal{N}}=\left\{\widetilde{\lambda}_{\varepsilon}^{(\theta)}\right\}^{\mathcal{N}} \rho_{\varepsilon}\left\{\widetilde{u}_{\varepsilon}^{(\theta)}\right\}^{\mathcal{N}}+\left\{f_{\varepsilon}^{(\theta)}\right\}^{\mathcal{N}} \quad n p u \quad x \in \Omega, \\
\left\{\widetilde{u}_{\varepsilon}^{(\theta)}\right\}^{\mathcal{N}}=0 \quad n p u \quad x \in \gamma_{\varepsilon}, \\
\frac{\partial\left\{\widetilde{u}_{\varepsilon}^{(\theta)}\right\}^{\mathcal{N}}}{\partial \nu}=0 \quad n p u \quad x \in \Gamma_{\varepsilon} \cup \Gamma_{1} \cup \Gamma_{2} \cup \Gamma_{3},
\end{gathered}
$$

где $\rho_{\varepsilon}$ задана формулой (1.2),

$$
\left\|\left\{f_{\varepsilon}^{(\theta)}\right\}^{\mathcal{N}}\right\|_{0}=O\left(\varepsilon^{\beta \mathcal{N}-4}\right)
$$




$$
\left\{\widetilde{u}_{\varepsilon}^{(\theta)}\right\}^{\mathcal{N}} \rightarrow u_{0}^{(\theta)} \quad \text { сильно в } L_{2}(\Omega) \text { при } \quad \varepsilon \rightarrow 0
$$

Напомним, что срезающая функция $\chi_{\beta, \Upsilon}(x)$ была введена перед леммой 3.11 . Этим мы завершили построение формальных асимптотик собственных значений и собственных функций.

Теперь перейдем к строгому обоснованию построенных асимптотик. Обоснование для случая кратного собственного значения имеет некоторые отличия от обоснования для случая простого собственного значения $\lambda_{0}$.

Учитывая, что $\left\|\left\{\widetilde{u}_{\varepsilon}^{(\theta)}\right\}^{\mathcal{N}}\right\|_{0} \rightarrow 1$ при $\varepsilon \rightarrow 0$, и применяя утверждение 1$)$ теоремы 2.3 для $\lambda=\widetilde{\lambda}_{\varepsilon}^{\mathcal{N}}, u=\widetilde{u}_{\varepsilon}^{\mathcal{N}}$ и $f=f_{\varepsilon}^{\mathcal{N}}$, заключаем, что

$$
\left|\left\{\widetilde{\lambda}_{\varepsilon}^{(\theta)}\right\}^{\mathcal{N}}-\lambda_{\varepsilon}^{(1)}\right|\left|\left\{\widetilde{\lambda}_{\varepsilon}^{(\theta)}\right\}^{\mathcal{N}}-\lambda_{\varepsilon}^{(2)}\right|=O\left(\varepsilon^{\beta \mathcal{N}-4}\right) .
$$

Это означает, что при любом фиксированном $\theta$ один из сомножителей в левой части имеет порядок $O\left(\varepsilon^{\frac{\beta \mathcal{N}-4}{2}}\right)$. Отсюда в силу произвольности в выборе $\mathcal{N}$ следует, что построенные асимптотические ряды $\widetilde{\lambda}_{\varepsilon}^{(1)}$ и $\widetilde{\lambda}_{\varepsilon}^{(2)}$ являются полными асимптотиками собственных значений $\lambda_{\varepsilon}^{(1)}$ и $\lambda_{\varepsilon}^{(2)}$ соответственно. Поскольку

$$
\left|\left\{\widetilde{\lambda}_{\varepsilon}^{(1)}\right\}^{\mathcal{N}}-\left\{\widetilde{\lambda}_{\varepsilon}^{(2)}\right\}^{\mathcal{N}}\right|>C \varepsilon
$$

то эти собственные значения различные (а следовательно, простые) и

$$
\left|\lambda_{\varepsilon}^{(1)}-\lambda_{\varepsilon}^{(2)}\right|>C \varepsilon
$$

где $C$ - постоянная.

Перейдем к обоснованию построенной асимптотики собственной функции. Из представления

$$
\left\{\widetilde{u}_{\varepsilon}^{(\theta)}\right\}^{\mathcal{N}}=u_{\varepsilon}^{(\theta)} \int_{\Omega} u_{\varepsilon}^{(\theta)}\left\{\widetilde{u}_{\varepsilon}^{(\theta)}\right\}^{\mathcal{N}} d x+\left\{u_{\varepsilon}^{(\theta)}\right\}^{\perp}, \quad\left\{u_{\varepsilon}^{(\theta)}\right\}^{\perp} \perp u_{\varepsilon}^{(\theta)}
$$

следует, что

$$
\left\{\widetilde{u}_{\varepsilon}^{(\theta)}\right\}^{\mathcal{N}}=\left\{T_{\varepsilon}^{(\theta)}\right\}^{\mathcal{N}} u_{\varepsilon}^{(\theta)}+\left\{u_{\varepsilon}^{(\theta)}\right\}^{\perp}, \quad\left\{T_{\varepsilon}^{(\theta)}\right\}^{\mathcal{N}}=1+o(1) .
$$

Из (4.46), (4.47) и леммы 4.1 следует, что функция $\left\{u_{\varepsilon}^{(\theta)}\right\}^{\perp}$ является решением краевой задачи

$$
\begin{gathered}
-\Delta\left\{u_{\varepsilon}^{(\theta)}\right\}^{\perp}=\widetilde{\lambda}_{\varepsilon}^{\mathcal{N}} \rho_{\varepsilon}\left\{u_{\varepsilon}^{(\theta)}\right\}^{\perp}+\left\{F_{\varepsilon}^{(\theta)}\right\}^{\mathcal{N}} \text { при } x \in \Omega, \\
\left\{u_{\varepsilon}^{(\theta)}\right\}^{\perp}=0 \text { при } x \in \gamma_{\varepsilon}, \\
\frac{\partial\left\{u_{\varepsilon}^{(\theta)}\right\}^{\perp}}{\partial \nu}=0 \text { при } x \in \Gamma_{\varepsilon} \cup \Gamma_{1} \cup \Gamma_{2} \cup \Gamma_{3},
\end{gathered}
$$


где неоднородность в уравнении имеет структуру, аналогичную (3.81), при этом

$$
\left\{F_{\varepsilon}^{(\theta)}\right\}^{\mathcal{N}}=O\left(\varepsilon^{\frac{\beta \mathcal{N}-4}{2}-m}\right) \text {. }
$$

Из (4.48), (4.49) и утверждения 2) теоремы 2.3 следует, что

$$
\left\|\left\{u_{\varepsilon}^{(\theta)}\right\}^{\perp}\right\|_{0}=O\left(\varepsilon^{\frac{\beta \mathcal{N}-4}{2}-m}\right) .
$$

И наконец, из $(4.47),(4.50)$ и произвольности в выборе $\mathcal{N}$ и $\Upsilon$ следует, что собственные функции имеют асимптотики (4.37), (4.38).

Теорема 4.1 доказана полностью.

Основная часть статьи была написана зимой 2003 г. во время пребывания автора в университете в г. Нарвик (Норвегия) и весной 2003 г. в университете Б. Паскаля в г. Клермон-Ферран (Франция). Автор выражает благодарность коллегам этих университетов за обеспечение замечательных условий для работы и поддержку. Выражаю признательность Р. Р. Гадыльшину за полезные обсуждения и ценные замечания.

\section{Список литературы}

1. Крылов A. Н. О некоторых дифференциальных уравнениях математической физики, имеющих приложения в технических вопросах // Изв. Николаевской морской академии. 1913. № 2. C. 325-348.

2. Тихонов A.Н., Самарский А. А. Уравнения математической физики. М.: Наука, 1972.

3. Sánchez-Palencia E. Perturbation of eigenvalues in thermoelasticity and vibration of system with concentrated masses // Trends and Application of pure Math. to Mechanics. Lecture notes in Physics. V. 195. Berlin: Springer-Verlag, 1984. P. 346-368.

4. Олейник О. А. Лекции по уравнениям с частными производными. М.: Изд-во МГУ, 1976.

5. Олейник O. А. О собственных колебаниях тел с концентрированными массами // Современные проблемы прикладной математики и математической физики. М.: Наука, 1988. C. $101-128$.

6. Олейник О.А. О спектрах некоторых сингулярно возмущенных операторов // УМН. 1987. T. 42. № 3. C. 221-222.

7. Oleinik O.A. Homogenization problems in elasticity. Spectrum of singularly perturbed operators // Non-classical continuum mechanics. Lecture Notes series. V. 122. Cambridge: Cambridge Univ. Press, 1987. P. 188-205.

8. Олейник O.A. О частотах собственных колебаний тел с концентрированными массами // Функциональные и численные методы математической физики. Киев: Наук. думка, 1988. C. $165-171$.

9. Головатый Ю. Д. Спектральные свойства колебательных систем с присоединенными массами: Дис. ... канд. физ.-мат. наук. М.: МГУ, 1988.

10. Головатый Ю.Д., Назаров С.А., Олейник О.А., Соболева Т.С. О собственных колебаниях струны с присоединенной массой // Сиб. матем. журн. 1988. Т. 29. № 5. C. $71-91$.

11. Олейник О.А., Соболева Т.С. О собственных колебаниях неоднородной струны с конечным числом присоединенных масс // УМН. 1988. Т. 43. № 4. С. 187-188.

12. Головатый Ю. Д. О собственных колебаниях и собственных частотах упругого стержня с присоединенной массой // УМН. 1988. Т. 43. № 4. С. 173-174.

13. Головатый Ю. Д. О собственных колебаниях и собственных частотах закрепленной пластинки с присоединенной массой // УМН. 1988. Т. 43. №5. С. 185-186. 
14. Nazarov S. A. Concentrated masses problems for a spatial elastic body // C. R. Acad. Sci. Paris. Ser. I. Math. 1993. V. 316. № 6. P. 627-632.

15. Argatov I. I., Nazarov S. A. Junction problem of shashlik (skewer) type // C.R. Acad. Sci. Paris Ser. I. Math. 1993. V. 316. № 12. P. 1329-1334.

16. Nazarov $S$. A. Interaction of concentrated masses in a harmonically oscillating spatial body with Neumann boundary conditions // RAIRO Model. Math. Anal. Numer. 1993. V. 27. № 6. P. $777-799$.

17. Головатый Ю. Д. Спектральные свойства колебательных систем с присоединенными массами: эффект локальных колебаний // Тр. ММО. 1992. Т. 54. С. 29-72.

18. Головатый Ю. Д. Спектральная задача Неймана для оператора Лапласа с сингулярно возмущенной плотностью // УМН. 1990. Т. 45. № 4. С. 147-148.

19. Назаров C.A. Об одной задаче Санчес-Паленсия с краевыми условиями Неймана // Изв. вузов. Математика. 1989. №11. С. 60-66.

20. Рахманов Н. У. О собственных колебаниях систем с концентрированными массами: Дис. ... канд. физ.-мат. наук. М.: МГУ, 1991.

21. Головатый Ю.Д., Назаров С.А., Олейник О.А. Асимптотика собственных значений и собственных функций в задачах о колебаниях среды с сингулярным возмущением плотности // УМН. 1988. Т. 43. № 5. С. 189-190.

22. Головатьй Ю. Д., Назаров С. А., Олейник О. А. Асимптотические разложения собственных значений и собственных функций задач о колебаниях среды с концентрированными возмущениями // Тр. Матем. ин-та им. В. А. Стеклова АН СССР. 1990. Т. 192. C. $42-60$.

23. Sánchez-Palencia E., Tchatat $H$. Vibration de systèmes elastiques avec masses concentrées // Rendiconti del Seminario matematico della Universita e politecnico di Torino. 1984. V. 42. № 3. P. 43-63.

24. Golovaty Y.D., Lavrenyuk A.S. Asymptotic expansions of local eigenvibrations for plate with density perturbed in neighbourhood of one-dimensional manifold // Mat. Stud. 2000. V. 13. № 1. P. 51-62.

25. Leal C., Sanchez-Hubert J. Perturbation of the Eigenvalue of a Membrane with a Concentrated Mass // Quarterly Appl. Math. 1989. V. XLVII. № 1. P. 93-103.

26. Lobo M., Pérez E. Asymptotic Behavior of the Vibrations of a Body Having Many Concentrated Masses Near the Boundary // C. R. Acad. Sci. Paris. Série II. 1992. V. 314. P. 13-18.

27. Lobo M., Pérez E. On Vibrations of a Body With Many Concentrated Masses Near the Boundary // Math. Models and Methods in Appl. Sci. 1993. V. 3. № 2. P. 249-273.

28. Lobo M., Pérez E. Vibrations of a Body With Many Concentrated Masses Near the Boundary: High Frequency Vibrations // Spectral Analysis of Complex Structures. Paris: Hermann, 1995. P. 85-101.

29. Lobo M., Pérez E. Vibrations of a Membrane With Many Concentrated Masses Near the Boundary // Math. Models and Methods in Appl. Sci. 1995. V. 5. № 5. P. 565-585.

30. Lobo M., Pérez E. High Frequency Vibrations in a Stiff Problem // Math. Models and Methods in Appl. Sci. 1997. V. 7. № 2. P. 291-311.

31. Lobo M., Pérez E. A Skin Effect for Systems with Many Concentrated Masses // C. R. Acad. Sci. Paris. Série IIb. 1999. V. 327. P. 771-776.

32. Gómez D., Lobo M., Pérez E. On the Eigenfunctions Associated with the High Frequencies in Systems with a Concentrated Mass // J. Math. Pures Appl. 1999. V. 78. P. 841-865.

33. Gómez D., Lobo M., Pérez E. On a Vibrating Plate with Concentrated Mass // C. R. Acad. Sci. Paris. Série IIb. 2000. V. 328. P. 494-500.

34. Lobo M., Pérez E. The skin effect in vibrating systems with many concentrated masses // Math. Methods Appl. Sci. 2001. V. 24. № 1. P. 59-80.

35. Oleinik O.A., Sanchez-Hubert J., Yosifian G.A. On vibration of membrane with concentrated masses // Bulletin des Sciences Mathematiques. 1991. V. 115. № 1. P. 1-27. 
36. Sanchez-Hubert J., Sánchez-Palencia E. Vibration and Coupling of Continuous System. Asymptotic methods. Berlin-Heidelberg: Springer-Verlag, 1989.

37. Sanchez-Hubert J. Perturbation des valeurs propres pour des systèmes avec masse concentrée // C. R. Acad. Sci. Paris Ser. II. Mec. Phys. Chim. Sci. Univers Sci. Terre. 1989. V. 309. № 6. Р. 507-510.

38. Доронина Е.И., Чечкин Г.А. О собственных колебаниях тела с большим количеством непериодически расположенных концентрированных масс // Тр. Матем. ин-та им. В. А. Стеклова РАН. 2002. Т. 236. С. $158-166$.

39. Rybalko $V$. Vibration of Elastic Systems with a Large Number of Tiny Heavy Inclusions // Asymptotic Analysis. 2002. V. 32. № 1. P. 27-62.

40. Chechkin G. A., Pérez E., Yablokova E. I. Non-Periodic Boundary Homogenization and "Light" Concentrated Masses // Indiana University Math. J. 2005. V. 54. № 2. P. 321-348.

41. Перес М.Е., Чечкин Г.А., Яблокова (Доронина) Е.И. О собственных колебаниях тела с "легкими" концентрированными массами на поверхности // УМН. 2002. Т. 57. № 6. C. $195-196$.

42. Чечкин $Г$. А. Об оценке решений краевых задач в областях с концентрированными массами, периодически расположенными вдоль границы. Случай “легких" масс // Матем. заметки. 2004. Т. 76. №6. С. 928-944.

43. Чечкин Г.А. О колебаниях тел с концентрированными массами, расположенными на границе // УМН. 1995. Т. 50. № 4. С. 105-106.

44. Чечкин Г.А. Граничное усреднение в областях с сингулярной плотностью // Дифф. уравн. 2003. Т. 39. №6. С. 855 .

45. Chechkin G. A. On vibration of partially fastened membrane with many "light" concentrated masses on the boundary // C. R. Méc. Acad. Sci. Paris. 2004. V. 332. № 12. P. 949-954.

46. Чечкин Г. А. Расщепление кратного собственного значения в задаче о концентрированных мaccax // УМН. 2004. Т. 59. № 4. С. 205-206.

47. Чечкин $Г$.А. Спектральные свойства эллиптической задачи с быстро осциллирующими граничными условиями // Краевые задачи для неклассических уравнений в частных производных. Новосибирск: ИМ СОАН СССР, 1989. С. 197-200.

48. Гадыльшин P. Р. Асимптотика собственного значения сингулярно возмущенной эллиптической задачи с малым параметром в граничном условии // Дифф. уравн. 1986. Т. 22. № 4. C. $640-652$.

49. Гадыльшин Р. Р. Расщепление кратного собственного значения задачи Дирихле для оператора Лапласа при сингулярном возмущении граничного условия // Матем. заметки. 1992. Т. 52. № 4. С. 42-55.

50. Планида М. Ю. О сходимости решений сингулярно возмущенных краевых задач для лапласиана // Матем. заметки. 2002. Т. 71. №6. С. 867-877.

51. Планида М. Ю. Асимптотика собственных значений для цилиндра, теплоизолированного на тонкой полоске // ЖВМиМФ. 2003. Т. 43. № 3. С. 403-413.

52. Борисов Д. И. О краевой задаче в цилиндре с частой сменой типа граничных условий // Матем. сб. 2002. Т. 193. № 7. С. 37-68.

53. Borisov D. I. On a model boundary value problem for Laplacian with frequently alternating type of boundary condition // Asymptotic Analysis. 2003. V. 35. № 1. P. 1-26.

54. Олейник О.А., Иосифьян Г. А., Шамаев А. С. Математические задачи теории сильно неоднородных упругих сред. М.: Изд-во МГУ, 1990.

55. Иосида К. Функциональный анализ. М.: Мир, 1967.

56. Чечкин Г. А. О краевых задачах для эллиптического уравнения второго порядка с осциллирующими граничными условиями // Неклассические дифференциальные уравнения в частных производных. Новосибирск: ИМ СОАН СССР, 1988. С. 95-104.

57. Соболев С. Л. Некоторые применения функционального анализа в математической физике. М.: Наука, 1988. 
58. Соболев С. Л. Избранные вопросы теории функциональных пространств и обобщенных функций. М.: Наука, 1989.

59. Ладыженская О.А., Уральщева Н. Н. Линейные и квазилинейные уравнения эллиптического типа. М.: Наука, 1977.

60. Лады женская О. А. Краевые задачи математической физики. М.: Наука, 1973.

61. Михайлов В. П. Дифференциальные уравнения в частных производных. М.: Наука, 1983.

62. Като Т. Теория возмущений линейных операторов. М.: Мир, 1972.

63. Ильин A. М. Краевая задача для эллиптического уравнения второго порядка в области с узкой щелью. І. Двумерный случай // Матем. сб. 1976. Т. 99 (141). С. 514-537.

64. Ильин A. М. Краевая задача для эллиптического уравнения второго порядка в области с узкой щелью. II. Область с малым отверстием // Матем. сб. 1977. Т. 103 (145). C. $265-284$.

65. Ильин А. М. Исследование асимптотики решения эллиптической краевой задачи в области с малым отверстием // Тр. сем. им. И. Г. Петровского. 1981. №6. С. 57-82.

66. Ильин $A M$. Согласование асимптотических разложений решений краевых задач. М.: Наука, 1989.

67. Чечкин $Г$. А. Усреднение краевых задач с сингулярным возмущением граничных условий // Матем. сб. 1993. Т. 184. №6. С. 99-150.

68. Gadyl'shin R. R. Asymptotics of the minimum eigenvalue for a circle with fast oscillating boundary conditions // C. R. Acad. Sci. Paris. Ser. I. 1996. V. 323. № 3. P. 319-323.

69. Гадыльшин P. P. О краевой задаче для лапласиана с быстро осциллирующими граничными условиями // Докл. РАН. 1998. Т. 362. № 4. С. 456-459.

70. Гадыльшин P. Р. Об асимптотике собственных значений для периодически закрепленной мембраны // Алгебра и анализ. 1998. Т. 10. № 1. С. 3-19.

71. Назаров С.A. Соединения сингулярно вырождающихся областей различных предельных размерностей. I // Тр. сем. И. Г. Петровского. 1995. Т. 18. С. 3-78.

72. Назаров С. А., Пламеневский Б. А. Эллиптические задачи в областях с кусочно гладкой границей. М.: Наука, 1991.

73. Борисов Д. И. Асимптотики и оценки собственных элементов лапласиана с частой непериодической сменой граничных условий // Изв. РАН. Сер. матем. 2003. Т. 67. № 6. C. $23-70$.

Московский государственный университет

Поступило в редакцию им. М. В. Ломоносова 02.06 .2004

E-mail: chechkin@mech.math.msu.su 